\title{
DT RESULTS OF TFTR'S ALPHA COLLECTOR
}

\author{
H. W. HERRMANN, S. J. ZWEBEN, D. S. DARROW, J. R. TIMBERLAKE \\ Princeton Plasma Physics Laboratory \\ Princeton, NJ 08543, USA
}

\author{
G. P. CHONG, A. A. HAASZ \\ University of Toronto, Institute for Aerospace Studies \\ Downsview, Ontario, Canada
}

C. S. PITCHER

Max-Planck-Institut fur Plasmaphysik

Garching bei Munchen, Germany

R. G. MACAULAY-NEWCOMBE

McMaster University, Dept. Engineering Physics

Hamilton, Ontario, Canada

\begin{abstract}
An escaping alpha collector probe has been developed for TFTR's DT phase to complement the results of the lost alpha scintillator detectors which have been operating on TFTR since 1988. Measurements of the energy distribution of escaping alphas have been made by measuring the range of alphas implanted into nickel foils located within the alpha collector. Exposed samples have been analyzed for 4 DT plasma discharges at plasma currents of 1.0 and 1.8 MA. The results at 1.0 MA are in good agreement with predictions for first orbit alpha loss at $3.5 \mathrm{MeV}$. The 1.8 MA results, however, indicate a large anomalous loss of partially thermalized alphas at an energy $\sim 30 \%$ below the birth energy and at a total fluence nearly an order of magnitude above expected first orbit loss. This anomalous loss is not observed with the lost alpha scintillator detectors in DT plasmas but does resemble the anomalous 'delayed' loss seen in DD plasmas. Several potential explanations for this loss process are examined. None of the candidate explanations proposed thus far are fully consistent with the anomalous loss observations.
\end{abstract}

\section{INTRODUCTION}

Present day

tokamaks

have begun to utilize the deuterium-tritium (DT) fusion reaction: $D+T \rightarrow \alpha(3.5 \mathrm{MeV})+n(14.1 \mathrm{MeV})$, making possible the experimental investigation of alpha particle behavior and its effect on thermonuclear plasmas. TFTR has conducted the first such systematic study of alpha particle physics. A crucial aspect of alpha particle physics is the fraction of alphas lost to the first wall. Alphas lost from the plasma prior to their thermalization reduce the self-heating power needed to achieve ignition. But more importantly, in the design of ITER and future DT reactors it will be necessary to predict the alpha particle losses to the first wall and divertor plates, since even a few percent loss may cause damage due to localized heating. Studies of alpha particle loss mechanisms could also prove valuable in developing much needed methods of $\mathrm{He}$ ash removal, burn control, and alpha channeling.

TFTR has previously relied on the lost alpha scintillator detectors $[1,2]$ as the sole escaping alpha diagnostic. In order to provide a complementary measurement of escaping alphas, a new alpha collector sample probe $[3,4]$ has been developed. The alpha collector probe operates on an entirely different physical principle, ie. the implantation and subsequent trapping of alpha particles in nickel foils [5]. The primary reasons this detection technique was selected are:

1. to improve the energy resolution with respect to the lost alpha scintillators

2. for its inherent ease of absolute calibration, allowing a valuable cross calibration for the lost alpha scintillators and validation of alpha loss models

3. for its immunity to high neutron fluxes, which may prove useful in ITER and future DT reactors.

The escaping alpha diagnostics (alpha collector probe and lost alpha scintillator detectors) and the conditions of the plasma discharges of interest are described in Section 2. Section 3 discusses the model used to predict first orbit alpha loss. In Section 4, the results of the alpha collector are compared to the first orbit loss model and the results of the lost alpha scintillators. Section 5 considers possible explanations for the anomalous results and in Section 6 a summary and conclusions are presented. 


\section{DISCLAIMER}

This report was prepared as an account of work sponsored by an agency of the United States Government. Neither the United Stales Government nor any agency thereof, sor any of their employees, makes any warranty, express or implied, or assumes any legal liability or responsibility for the accuracy, completeress, or usefulness of any information, apparatus, product, or process disclosed, or represents that its use would not infringe privately owned rights. Reference herein to any specific commercial product, process, or service by trade name, trademark, manufacturer, or otherwise does not necessarily constitute or imply its endorsement, recommendation, or favoring by the United States Government or any agency thereof. The views and opinions of authors expressed herein do not necessarily state or reflect those of the United States Governmeat or any agency thereof. 


\section{DISCLAIMER}

Portions of this document may be illegible in electronic image products. Images are produced from the best available original document. 


\section{THE ESCAPING ALPHA DIAGNOSTICS}

While the focus of this study is alpha loss observations of the alpha collector probe, it is useful to compare these observations to those of the lost alpha scintillator detectors. It is therefore necessary to compare the detection techniques and relative locations of the various detectors. Section 2.1 covers the details of the alpha collector including its positioning relative to the nearest scintillator detector, and the conditions under which the exposures were conducted. Section 2.2 presents a brief description of the lost alpha scintillator detectors.

\subsection{Alpha Collector Probe}

\subsubsection{Detection Technique}

The alpha collector probe $[3,4]$ is based on the foil deposition technique originally proposed by Langley [5] and a similar method used on JET to determine the energy distribution of He- 3 ions accelerated by ICRH $[6,7]$. In the TFTR implementation of this technique, escaping alpha particles whose trajectories intercept the detector are implanted into a stack of nickel foils consisting of ten layers of $1 \mu \mathrm{m}$ thick foil. This is sufficient to stop DT fusion alphas at $3.5 \mathrm{MeV}$, which have an expected penetration range of $\sim 6 \mu \mathrm{m}$ in nickel [8]. The alpha particles form a distribution of implantation ranges in the foil stack that is dependent on their incident angles and energies. Once the alphas are stopped in the $\mathrm{Ni}$, they are trapped and remain immobile as long as the Ni remains below a critical temperature of $\sim 400^{\circ} \mathrm{C}$ [9].

The foils are removed from the TFTR vacuum vessel after exposure to the alpha flux of one or more discharges. They are then analyzed for He content by melting the foils one at a time in an off-site vacuum chamber (located at the University of Toronto), thus releasing the $\mathrm{He}$, and measuring the partial pressure of $\mathrm{He}$ with a Residual Gas Analyzer $[3,10]$. The alpha energy spectrum is then inferred from the range distribution of $\mathrm{He}$ in the $\mathrm{Ni}$ foil stack. Calibration implants of known energies and total fluences from a Van de Graaff accelerator and from a sealed Am241 alpha source have been used to check the accuracy of the implantation model (see section 3 ) and sample analysis method [10].

\subsubsection{TFTR Geometry}

Fig. 1 depicts a poloidal cross section of TFTR showing the exposure position of the alpha collector probe at the bottom of the vessel, and the poloidal projection of the RF limiters. TFTR has nine carbon poloidal RF limiters of varying poloidal extent to protect the RF launchers. They

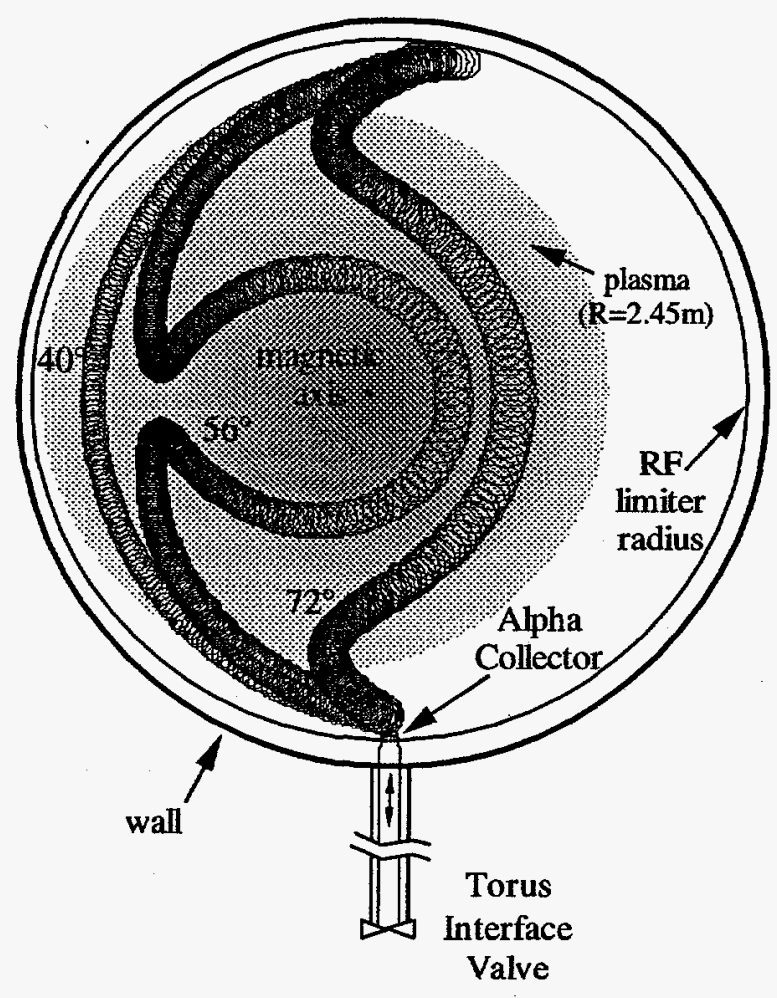

FIG. 1. Poloidal cross section of TFTR illustrating a co-going passing particle $\left(\chi_{t}=40^{\circ}\right.$, where $\chi_{t}$ is the toroidal pitch angle at the detector), a trapped particle at the fattest banana orbit $\left(\chi_{t}=56^{\circ}\right)$, and a deeply trapped orbit $\left(\chi_{t}=72^{\circ}\right)$ that strike the alpha collector probe located at the bottom of the vessel for a 1.8 MA plasma. Once exposed, the probe is lowered below the torus interface valve so it can be isolated and removed.

are centered at a major radius of $261 \mathrm{~cm}$ and have a minor radius of $99 \mathrm{~cm}$.

After exposure to one or more discharges, the probe is lowered remotely and isolated from the TFTR vacuum vessel by shutting the torus interface valve shown in Fig. 1. The probe chamber can then be vented and the probe head removed through a six inch conflat flange. The exposed nickel foils are then removed for analysis [10] and replaced with new foils. Also shown in Fig. 1 are various alpha particle orbits that strike the detector for a $2.45 \mathrm{~m}$ major radius plasma with a plasma current of 1.8 MA. These orbits will be discussed in more detail in section 3.

Fig. 2 is a partial toroidal cross section of TFTR showing the position of the alpha collector with respect to the nearby poloidal RF limiters and the $90^{\circ}$ lost alpha scintillator detector. The alpha collector and the lost alpha scintillator are located in adjacent bays separated by one of the $20 \mathrm{TF}$ field coils, placing them $18^{\circ}$ apart toroidally. The major radii of the alpha collector probe and the $90^{\circ} \mathrm{scin}-$ tillator detector are $262.5 \mathrm{~cm}$ and $259.2 \mathrm{~cm}$ respectively, 


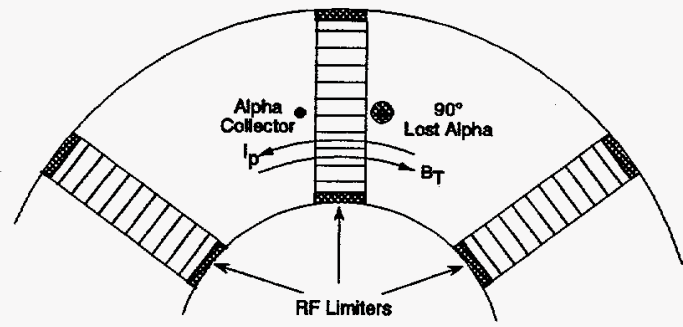

FIG. 2. Top view of TFTR vessel showing the relative locations of the alpha collector and the $90^{\circ}$ lost alpha scintillator detector with respect to the nearest $R F$ limiters.

placing each of them within $2 \mathrm{~cm}$ of the major radius of the RF limiter center.

Particles intercepting the $90^{\circ}$ lost alpha scintillator detector on co-going (in relation to the plasma current) orbits have $45^{\circ}$ of toroidal clearance between the center of the nearest $\mathrm{RF}$ limiter and the detector. Particles intercepting the alpha collector on co-going orbits, however, only have $9^{\circ}$ of toroidal clearance, making it necessary to position the probe closer to the plasma to avoid shadowing of these orbits by the limiter. This is essential for detection of first orbit loss since the majority of this loss occurs on the co-going leg of trapped banana orbits such as the $56^{\circ}$ and $72^{\circ}$ orbits depicted in Fig. 1. Orbits near the $56^{\circ}$ 'fattest banana' dominate first orbit loss because they pass closest to the magnetic axis where the alpha source rate is peaked.

Fig. 3 shows the alpha collector and nearest RF limiter as seen when looking toward the center of the torus. This figure depicts a co-going alpha particle orbit entering a collimating port on the detector. Alpha particles can enter any one of a series of 16 collimating ports that are separated into two rows on the cylindrical probe head. At the back of each port is the 10 layer stack of $1 \mu$ m thick nickel foils into which the alpha particles implant. Each port only accepts particles within a particular range of pitch angles.

The edge of the probe and the RF limiter are separated toroidally by $12.4 \mathrm{~cm}$. The probe was placed in the same position for each exposure, placing the center of the upper row of collimating ports $\sim 0.5 \mathrm{~cm}$ radially inward from (ie. above) the nearest limiter, and the lower row $\sim 0.6 \mathrm{~cm}$ radially outward from (ie. below) the limiter. This was sufficient to avoid shadowing of first orbit loss at $3.5 \mathrm{MeV}$ to both rows, as will be shown in section 4.4. The top of the lower row of collimating ports was only $\sim 0.3 \mathrm{~cm}$ below the RF limiter. The larger toroidal separation for co-going orbits between the nearest RF limiter and the $90^{\circ}$ scintillator detector allows its $0.1 \mathrm{~cm}$ high pinhole aperture to be placed $\sim 1.2 \mathrm{~cm}$ below the RF limiter [2], or $\sim 0.9 \mathrm{~cm}$

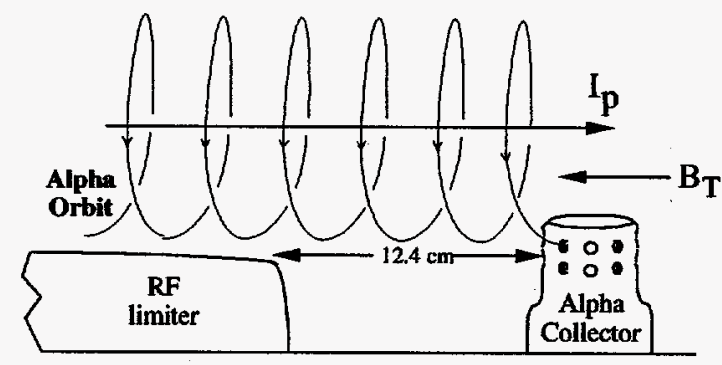

FIG. 3. Side view (looking in toward centerline of TFTR) of the alpha collector probe head depicting a co-going alpha particle entering one of the 16 collimating ports.

below the top of the lower row of the alpha collector.

\subsubsection{Probe Head Design}

Two separate alpha collector probe head designs were used. A cross section taken through the middle of a row of collimating ports is shown in Fig. 4(a) for the original design and Fig. 4(b) for the redesigned probe head. In the original design, the $\mathrm{Ni}$ foil is wrapped around a $3.175 \mathrm{~cm}$ diameter graphite cylindrical spool that fits inside the carbon-fiber-composite outer shell of the probe head. The original design has ports of $0.635 \mathrm{~cm}$ in width $(w)$ and depth $(d)$, whereas the redesign has $w=0.635 \mathrm{~cm}$, but has twice the port depth at $d=1.27 \mathrm{~cm}$. These port dimensions allow high energy particles with trajectories within $\pm 45^{\circ}$ of a port's axis to strike the foils in the original design, but an acceptance range of only $\sim \pm 27^{\circ}$ for the redesign, which improved the pitch angle resolution.

The Ni foil, acquired from Goodfellow Corporation, has a $99.95 \%$ purity rating and a $\pm 10 \%$ thickness accuracy as determined by weight. A foil stack is prepared by folding a $10 \mathrm{~cm} \mathrm{x} 10 \mathrm{~cm}$ sheet of $1 \mu \mathrm{m}$ Ni foil 9 times to form a $10 \mathrm{~cm} \times 1 \mathrm{~cm}$ strip consisting of 10 layers. The foil is folded in a 'rolled' fashion, rather than an 'accordion' fashion, to limit the amount of tritium that can diffuse to the inner layers during exposure to a D-T plasma. Tritium adhering to the foil surfaces presents a radioactive contamination problem once the foils are removed from the probe. The first layer is discarded (with the exception of exposure C) since it is directly exposed to tritium in the vessel, and the remaining layers are decontaminated with a wet argon flow [10].

The Ni foil stack is wrapped around and 'sewn' onto the spool using $0.25 \mathrm{~mm}$ aluminum wire and the overlapping ends of the $\mathrm{Ni}$ are spot welded together. The $\mathrm{Al}$ wire holds the Ni tightly to the graphite spool, improving the thermal contact between the $\mathrm{Ni}$ and the spool allowing the spool to act as a heat sink. The $660^{\circ} \mathrm{C}$ melting point of $\mathrm{Al}$ enables the wire to also act as a temperature indicator. If the $\mathrm{Al}$ wire exposed in a collimating port experiences any 

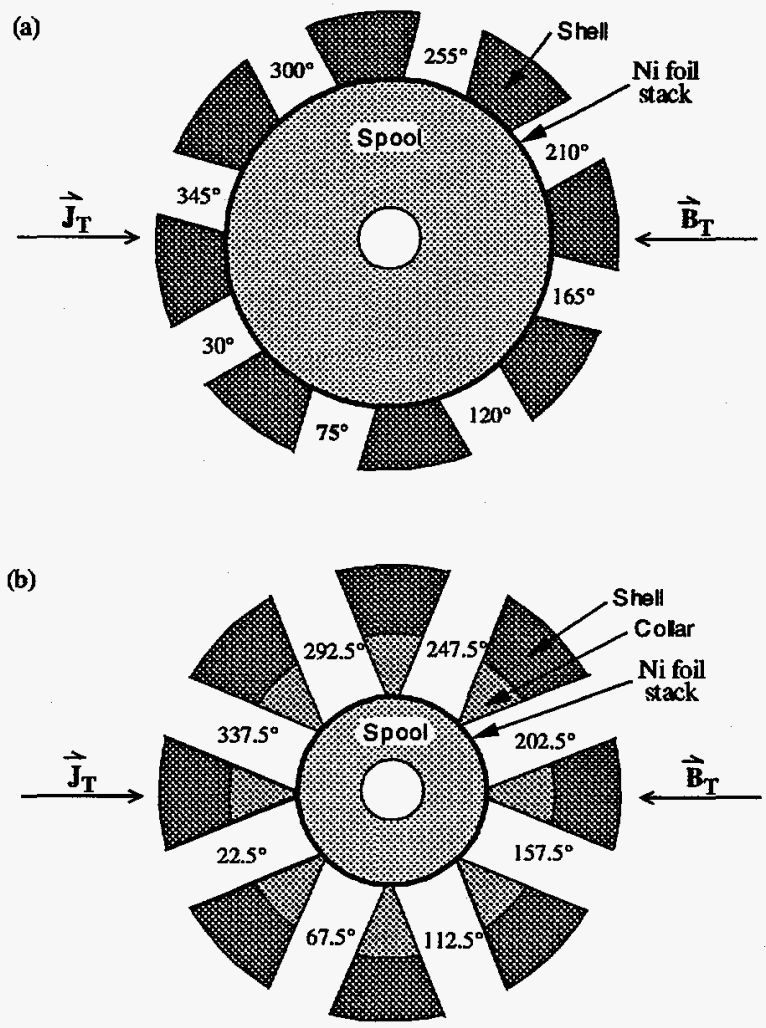

FIG. 4. Midplane cross section of a row of collimating ports for the $(a)$ original design and $(b)$ redesigned alpha collector probe head. The stack of 10 layers of $1 \mu \mathrm{m} \mathrm{Ni}$ foil is wrapped onto the inner spool which is then inserted into an outer shell. Cylindrical $0.635 \mathrm{~cm}$ diameter holes drilled into the $0.635 \mathrm{~cm}$ thick outer shell make up the collimating ports. The collimator depth is extended to $1.27 \mathrm{~cm}$ in the redesigned head of $(b)$ by inserting a cylindrical $0.635 \mathrm{~cm}$ thick collar that also has $0.635 \mathrm{~cm}$ diameter holes drilled into it. The spool diameters are (a) $3.175 \mathrm{~cm}$ and (b) $1.905 \mathrm{~cm}$

melting due to heat flux from a plasma discharge then it is assumed that the $\mathrm{Ni}$ foil in that port exceeded $400^{\circ} \mathrm{C}$ and the implanted He sample is no longer a reliable indicator of escaping alphas.

Each row of collimating ports has 8 evenly spaced ports placed $45^{\circ}$ apart. The orientation of each port is labeled in Fig. 4 with respect to the toroidal direction $\left(0^{\circ}\right.$ corresponding to the collection of co-going particles). The redesigned head was rotated with respect to the original probe head by $7.5^{\circ}$ clockwise when looking down on it. This was to line it up with the scintillator detector which is oriented along $67.5^{\circ}$. For convenience, the orientations of the original design will be referred to when discussing the probe in general (subtract $7.5^{\circ}$ to get the redesign orientations).

\subsubsection{DT Exposures}

The alpha collector has been exposed to a total of 5 DT discharges. Plasma parameters for these 5 discharges, labeled A-E, are given in Table I. These discharges were conducted at low neutral beam injection (NBI) power to avoid MHD activity, so that first orbit loss was expected to be the dominant loss mechanism. The power was also kept low to avoid probe overheating, although thermal design considerations does allow its use in high power discharges with plasmas as large as $R=2.52 \mathrm{~m}$.

Exposure A, which was actually exposed to 2 identical discharges, resulted in melting of a majority of the foils and $\mathrm{Al}$ wires, possibly due to excessive beam ion loss [3]. The exposure A foils were not analyzed for He content due to the excessive heat damage. Beam ion loss is highly dependent on the direction of beam injection. For example, the calculated prompt first orbit loss of neutral beam ions ranges from 30 to $38 \%$ (depending on the beam tangency radius) for counter-injection, and only 0 to $1 \%$ for co-injection, for a $2.6 \mathrm{~m}$ major radius plasma at a plasma current of 0.9 MA [11]. To reduce the potential for beam ion loss, all subsequent exposures were conducted using co-going only NBI.

Exposures B through E suffered only minimal foil heat damage, and only in ports that had a direct line of sight to the magnetic field on the side opposite the nearest RF limiter. This corresponds to ports at $165^{\circ}$ and $210^{\circ}$. Thermal plasma could flow along field lines unimpeded to the foils in these ports. These four samples (ie. the upper and lower row of the $165^{\circ}$ and $210^{\circ}$ ports) were not analyzed for this reason.

Exposures D and E made use of the redesigned probe head. To minimize overheating of foil samples, the redesign incorporated the deeper collimating ports that could exclude small gyroradius beam ion loss to the foils in the $67.5^{\circ}$ port through better collimation, while still allowing large gyroradius fusion produced alphas to implant into these foils [3]. The redesign has not been evaluated during the use of counter-going beams to see if foil heat damage can be avoided.

\subsection{Lost Alpha Scintillator Detectors}

Four scintillator detectors are installed on TFTR to detect fusion product losses to the wall $[1,2]$. While these detectors were designed to detect alpha particles, they are also capable of detecting the 'alpha-like' D-D fusion products (ie. $3 \mathrm{MeV}$ proton and $1 \mathrm{MeV}$ triton). These detectors are installed at various poloidal angles below the outer midplane $\left(20^{\circ}, 45^{\circ}, 60^{\circ}\right.$, and $\left.90^{\circ}\right)$ and are all at the same toroidal angle. As illustrated in Fig. 5 each 
TABLE I. PARAMETER LIST

\begin{tabular}{lccccc}
\hline Exposure & $\mathrm{A}$ & $\mathrm{B}$ & $\mathrm{C}$ & $\mathrm{D}$ & $\mathrm{E}$ \\
\hline Probe head & Original & Original & Original & Redesign & Redesign \\
Shot \# & $73319 / 73320$ & 74796 & 76014 & 80553 & 84593 \\
$\mathrm{I}_{\mathrm{p}}$ (MA) & 0.6 & 1.8 & 1.0 & 1.8 & 1.0 \\
$\mathrm{R}_{0}(\mathrm{~m})$ & 2.45 & 2.45 & 2.45 & 2.45 & 2.45 \\
$\mathrm{~B}_{\mathrm{t}}(\mathrm{Tesla})$ & 4.75 & 4.75 & 4.88 & 4.87 & 4.88 \\
$\mathrm{P}_{\mathrm{b}}(\mathrm{MW})$ & 5.2 & 10.2 & 9.4 & 12.7 & 11.1 \\
$\mathrm{t}_{\mathrm{b}}(\mathrm{s})$ & $3.0-3.7$ & $3.0-3.7$ & $3.0-3.7$ & $3.0-4.0$ & $3.0-3.7$ \\
$\mathrm{~S}_{\mathrm{n}}\left(\mathrm{x} 10^{17} \mathrm{n}\right)$ & 0.6 & 1.3 & 1.1 & 4.3 & 1.8 \\
$\mathrm{n}_{\mathrm{e} 0}\left(\mathrm{x} 10^{13} \mathrm{~cm}^{-3}\right)$ & --- & 4.6 & 3.1 & 4.2 & 3.6 \\
$\mathrm{~T}_{\mathrm{e} 0}(\mathrm{keV})$ & --- & 6.1 & 5.8 & 7.4 & 6.4 \\
$\tau_{\mathrm{sd} 0}(\mathrm{~s})$ & --- & 0.21 & 0.29 & 0.30 & 0.28 \\
\hline
\end{tabular}

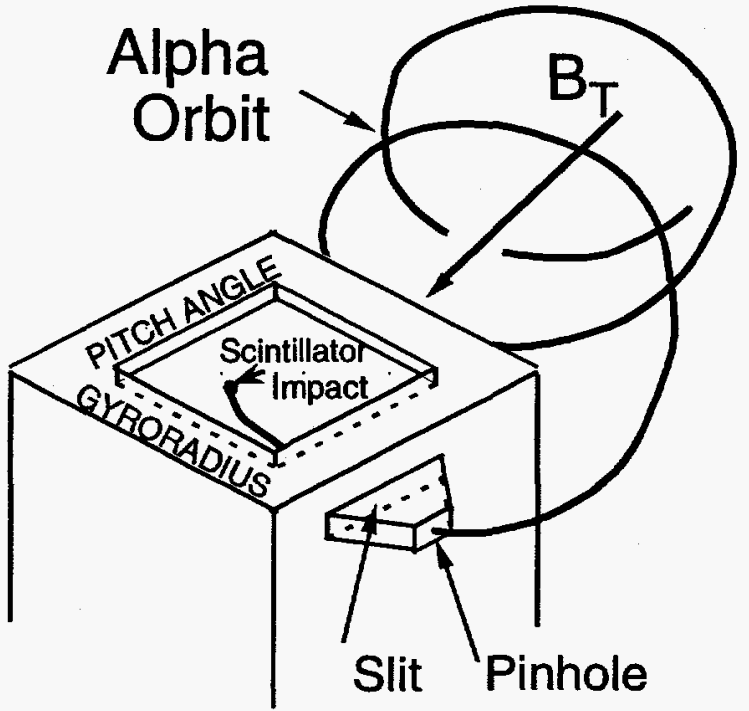

FIG. 5. Schematic diagram of the lost alpha scintillator detector located $90^{\circ}$ below the outer midplane. The escaping alphas enter a pair of apertures that disperse them in pitch angle and gyroradius. The 2-D image of the visible light emission from the scintillation screen is transmitted through a quartz fibreoptic bundle to a gated intensified video camera for analysis.

detector consists of a pinhole and slit collimator designed to disperse fusion products along a rectangular scintillator according to gyroradius $\rho$ (depending on their energy) in one dimension and pitch angle $\chi$ (depending on their magnetic moment) in the other. The visible light from ion impacts on the scintillator is imaged onto a shielded CCD camera and a series of photo multiplier tubes (PMT's). A detector analysis code determines a $(\rho, \chi)$ grid that is used to interpret the camera images. For this grid the $\rho$ coordi- nate is the centroid of the predicted scintillator impacts for an ion of gyroradius $\rho_{0}$ (the gyroradius the ion would have if all its energy were put into perpendicular motion, ie. at $\chi=90^{\circ}$ ) and the $\chi$ coordinate is the orbit's toroidal pitch angle $\chi_{t}$ measured locally with respect to the cogoing toroidal field direction at the detector. Specifics on the design and use of the lost alpha scintillators can be found in Refs. [1,2].

The alpha collector results are compared to measurements made with the lost alpha scintillator detector located $90^{\circ}$ below the outer midplane (ie. at the bottom of the vessel where the alpha collector is also located). Although these two probes are close to one another (toroidally separated by $\sim 83 \mathrm{~cm}$ ), the proximity of RF limiters capable of shadowing the detectors from alpha loss is different (see section 2.1.2).

\section{FIRST ORBIT LOSS MODEL}

First orbit loss is the loss associated with particles born on orbits that intersect the wall on their first bounce (ie. before completing a poloidal transit). These particles are lost with very nearly their birth energy since the time for one bounce $(<10 \mu \mathrm{sec})$ is much less than the collisional slowing down time $\left(\tau_{s d} \approx 200 \mathrm{~ms}\right.$ energy efolding time) [12]. So the energy of first orbit lost particles should be $\sim 3.5 \mathrm{MeV}$ with a Doppler spread of up to $\sim \pm 0.5 \mathrm{MeV}$ caused by the beam-target and beam-beam reactions $[1,13]$. This prompt loss follows the neutron signal very closely in time since for each neutron produced by fusion, an alpha is also produced. The global fraction of particles that are first orbit lost decreases with increas- 
ing plasma current. This is due to the reduced banana widths of trapped particles at higher current, resulting in a particle staying closer to a given flux surface and thus farther from the walls.

\subsection{Pitch Angle Distribution}

First orbit loss can be calculated using the PPPL's Lorentz ORBIT code [14]. The Lorentz ORBIT code integrates the Lorentz force equation to trace a single charged particle's trajectory. Fig. 1 shows some typical orbits at various pitch angles that strike the alpha collector. The orbit at a pitch angle of $40^{\circ}$ is a co-going passing orbit, whereas the other two orbits are trapped orbits. The orbit at a pitch angle of $56^{\circ}$ corresponds to the fattest banana orbit (ie. at the passing-trapped boundary) and passes closest to the magnetic axis where the alpha source profile is peaked, thus giving the largest contribution to first orbit loss.

By integrating the source profile along particle orbits backwards in time from the detector through the plasma and taking into account the detector area and solid angle, the code calculates the expected collection fraction (alphas/neutron) and pitch angle distribution of first orbit loss striking the detector. The collection fraction is simply the fraction of the total alpha production (equal to neutron production) that is collected by a foil stack inside one of the collimating ports and can be on the order of $10^{-6}$ alphas/neutron as calculated by ORBIT.

This collection fraction can be estimated by multiplying the expected global first orbit loss fraction by the ratio of the area of the vessel wall that alpha particles strike to the area of the exposed foil in the detector. For a 10\% global first orbit loss spread approximately evenly over the bottom $\sim 1 / 3$ (ion grad $B$ drift direction is down) of the $\sim 100 \mathrm{~m}^{2}$ vessel wall it would be expected that an area the size of the exposed foil surface of $\sim 3 \times 10^{-5} \mathrm{~m}^{2}$ would collect $\sim 1 \times 10^{-7}$ of the lost alphas. However, since the foils are perpendicular to the wall they intercept particles that would have struck the wall over a larger area than the foil area, giving the foils a projected area onto the wall about an order of magnitude larger than their geometrical area. This raises the estimate based on the detector to wall area to $\sim 10^{-6}$ consistent with the code results.

Fig. 6 is a plot of the predicted alpha collection fraction for first orbit loss as a function of collimating port orientation for the 1.8 MA exposures calculated using the Lorentz ORBIT code. The squares on the exposure B prediction mark the port orientations of the original probe head design which was used for this shot. Likewise, the circles on the exposure D prediction mark the port orientations of the redesigned probe head. The alpha collec-

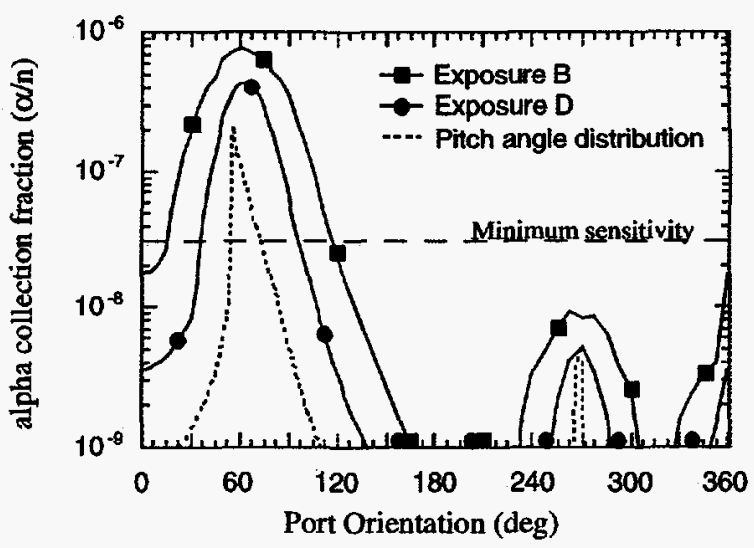

FIG. 6. Predicted alpha collection fraction (alphas/neutron) as a function of port orientation for the $1.8 \mathrm{MA}$ exposures using the original (exposure $B$ ) and the redesigned probe head (exposure D). The dashed curve represents the expected pitch angle distribution in arbitrary units. The horizontal dashed line at $3 \times 10^{-8}$ alphas/neutron represents the minimum sensitivity assuming $1 \times 10^{17}$ global neutron production.

tion fractions are the detector responses to the local pitch angle distribution of the expected first orbit loss, which is plotted in arbitrary units (dashed curve) for comparison. The peaks of the detector response curves (B \& D) are shifted to higher pitch angle with respect to the local pitch angle distribution due to the asymmetry in this distribution (ie. the high pitch angle tail). The reduced magnitude (by $\sim 45 \%$ at the $60^{\circ}$ peak) of the exposure $D$ curve with respect to the exposure $B$ curve in Fig. 6 is the result of reduced alpha collection associated with the deeper collimating ports of the redesigned head [3].

Since the alpha particles of interest have gyroradii much larger than the dimensions of the collimating port $(\rho \approx 5 \mathrm{~cm} \gg 0.64 \mathrm{~cm} \approx w)$, their paths inside the port can essentially be thought of as straight lines. Thus an alpha striking the foil surface in the original probe head design, in which the port's depth $(d)$ and width $(w)$ are equal $(0.635 \mathrm{~cm})$, can have a maximum angle of incidence, $\alpha_{\max }=\tan ^{-1}(w / d)$, of $45^{\circ}$. This maximum angle of incidence translates to $\pm 45^{\circ}$ pitch angle acceptance range about the orientation of the collimating port axis. The maximum angle of incidence for the redesigned head, in which $d=2 w$, is $26.6^{\circ}$. The FWHM of the detector response curves in Fig. 6 can be shown to be approximately the FWHM of the local pitch angle distribution $\left(\sim 5^{\circ}\right)$ plus half of the pitch angle acceptance range of the collimating port used in each design $\left(45^{\circ}\right.$ for the original head; $\sim 27^{\circ}$ for the redesign).

The detector response for inboard facing (toward the centerline of the torus, ie. $180^{\circ}-360^{\circ}$ ) ports in Fig. 6 lie below the minimum sensitivity, illustrated by the horizontal dashed line, estimated for a $1 \times 10^{17}$ total neutron 
production (for increased neutron production, the minimum sensitivity in alpha collection fraction is reduced). This is because an alpha particle undergoing left handed gyro-motion about the magnetic field (see Fig. 3) must approach the probe head from beneath in order to enter an inboard port and is thus much more likely to be scraped off by the RF limiter before reaching the probe. Only particles with a pitch angle very close to $90^{\circ}$ can intercept the inboard side of the detector since they travel nearly straight down (eg. ripple well trapped particles) and can avoid limiter scrape off. These particles are represented by the peaks centered at the $270^{\circ}$ port position. However, these particles would most likely be shadowed by the probe head itself before they could enter a collimating port, an effect that is not taken into account in this simulation. Thus it is expected that inboard facing ports would not collect significant quantities of escaping alphas.

Since an alpha is produced for each neutron, the calculated collection fraction is converted to expected alpha fluence by multiplying by the global neutron production. The code's accuracy is highly dependent on the assumed source and current profiles as is discussed in section 4.5. These profiles are generally obtained from the time dependent transport code, TRANSP [15], which takes inputs from various diagnostics to generate time dependent plasma parameters.

\subsection{Range Distribution}

The Lorentz ORBIT code predicts the total fluence and pitch angle distribution to a detector. However, to determine the range distribution of alphas in the nickel foils it is necessary to determine the angle of incidence distribution of alphas on the foils. The conversion from pitch angle distribution to incident angle distribution is accomplished using an auxiliary code called PORT developed specifically for this detector. PORT launches particles from a grid on the foil surface of each port at various pitch angles and gyro-phases, weighted by the ORBIT-calculated pitch angle distribution. Particle drifts, such as grad $B$ and curvature drift, are ignored since the particles are generally tracked for less than one gyro-orbit to determine if they clear the probe head. Thus a particle orbit is a simple helix about the magnetic field vector, defined by the particle's gyroradius $\rho$ and pitch angle, $\chi=\arccos \left(v_{\|} / v\right)$, where $v$ is the particle's velocity and $v_{\|}$is the parallel velocity component along the magnetic field. The magnetic field at the probe location (ie. at the bottom of vessel) is assumed to lie in the horizontal plane (ie. parallel to the midplane) and to make an angle $\chi_{b t}$ with the toroidal direction determined by $\chi_{b t}=\arctan \left(B_{p} / B_{t}\right)$, where $B_{p}$ is the poloidal field and $B_{t}$ is the toroidal field
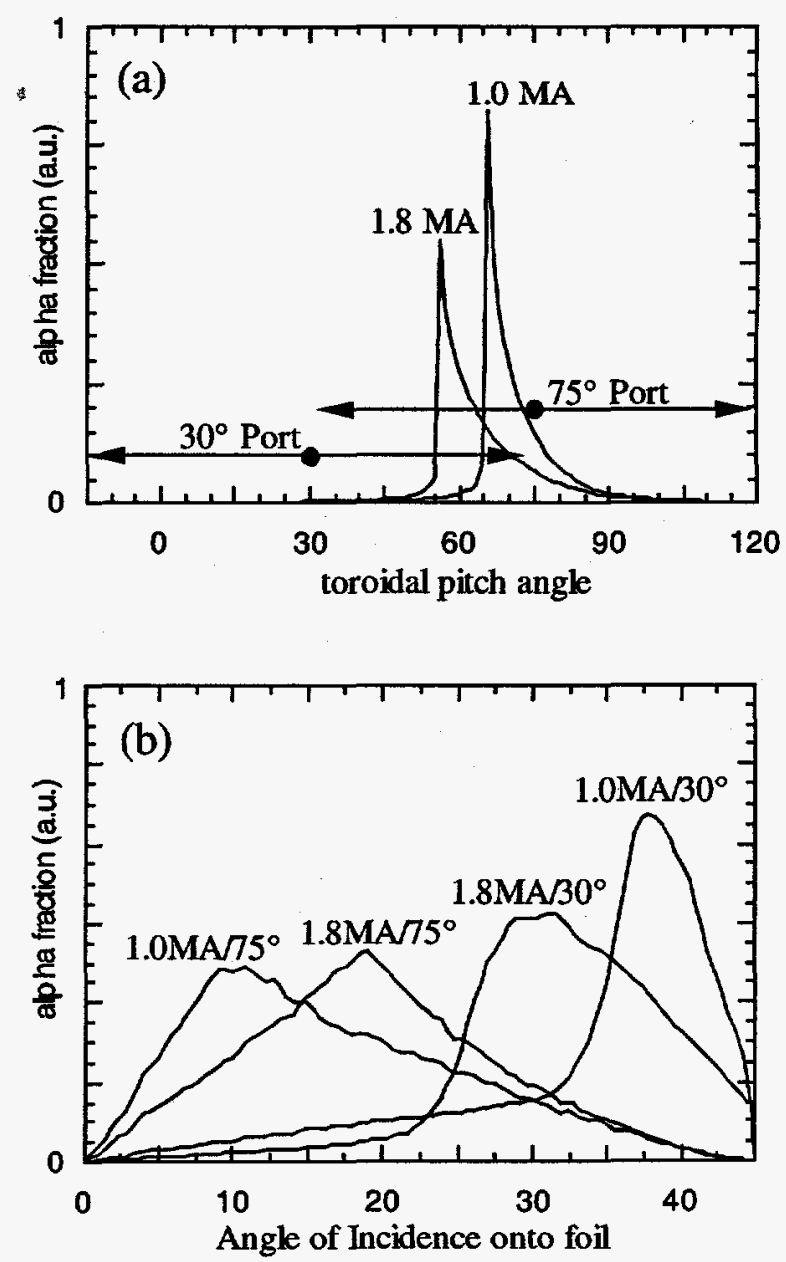

FIG. 7. (a) Toroidal pitch angle distributions in arbitrary units calculated by the Lorentz ORBIT code for $3.5 \mathrm{MeV}$ alphas from exposures $B(1.8 \mathrm{MA})$ and $C(1.0 \mathrm{MA})$. The $\pm 45^{\circ}$ acceptance range of the original probe design is depicted for the $30^{\circ}$ and $75^{\circ}$ ports. (b) The resulting angle of incidence distributions for the $30^{\circ}$ and $75^{\circ}$ ports calculated by PORT for the pitch angle distributions shown in (a).

at the alpha collector. PORT assumes that the first orbit loss to a detector port is independent of gyro-phase (good to within $\sim 20 \%$ as determined by ORBIT) and that the foil surface is flat. If a particle clears the port walls and the probe head, it is counted and its angle of incidence is determined. The angle of incidence, $\alpha\left(0^{\circ}\right.$ corresponding to normal incidence), is determined by taking the scalar product between the particle's velocity vector at the foil surface and a unit vector along the axis of the collimating port and is found to be:

$\cos \alpha=\cos \chi \cos \chi_{0}+\sin \chi \sin \chi_{0} \cos \gamma$

where $\chi$ is the particle's local pitch angle $\left(0^{\circ}\right.$ being along the magnetic field in the direction of the plasma current), $\chi_{0}$ is the angle between the magnetic field and the axis 

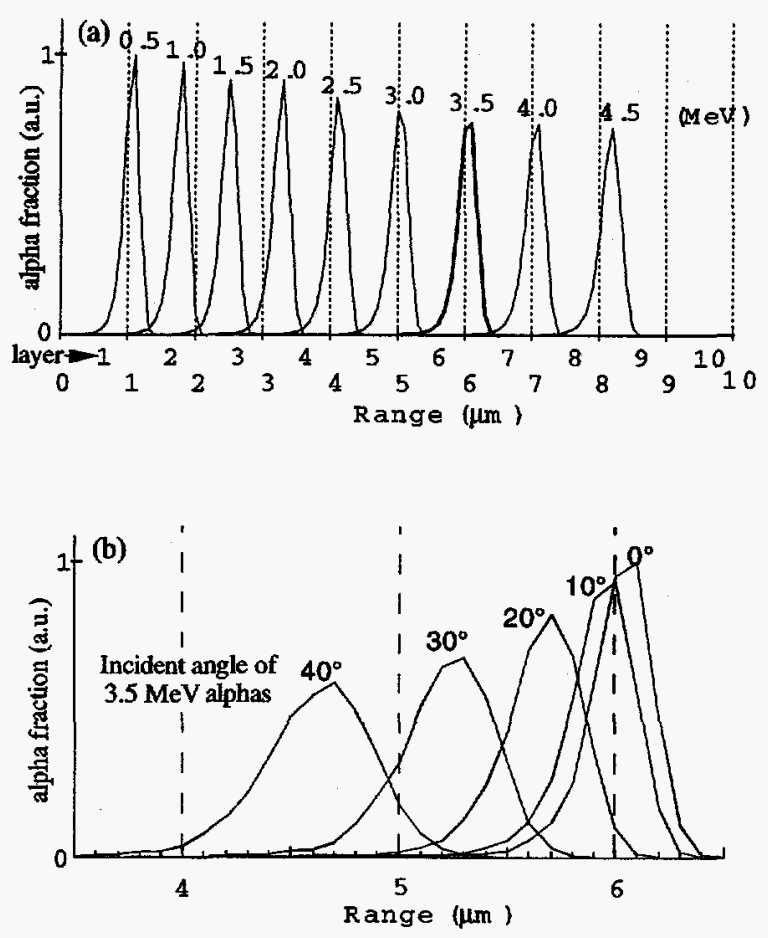

FIG. 8. (a) Implantation range distributions for monoenergetic alpha particles implanted into nickel at various energies and at normal incidence as calculated by the TRIM-95 code. The boundaries between the 1 um thick foil layers are depicted by the dashed lines. (b) Implantation range distributions for $3.5 \mathrm{MeV}$ alpha particles implanted into nickel at various angles of incidence.

of the collimating port, and $\gamma$ is the gyro-phase of the particle at the foil $\left(0^{\circ}\right.$ corresponding to the bottom of a gyro-orbit). Fig. 7 shows the conversion of 1.0 MA and 1.8 MA ORBIT calculated pitch angle distributions for $3.5 \mathrm{MeV}$ alphas to incident angle distributions for a $30^{\circ}$ and a $75^{\circ}$ port using PORT. Notice that the $1.8 \mathrm{MA}$ pitch angle distribution of Fig. 7 (a) has a peak at $56^{\circ}$ corresponding to the fattest banana orbit, which was depicted in Fig. 1, since it passes closest to the magnetic axis where the source profile is peaked.

Once the distribution of incident angles onto the foil is determined using the PORT code, the range distribution of $3.5 \mathrm{MeV}$ first orbit lost alphas can be determined using IBM's TRIM-95 code [8]. The TRIM code uses a Monte Carlo algorithm to calculate the penetration of ions into solids. Fig. 8(a) shows the TRIM calculated range distributions for $\mathrm{He}$ ions at various energies into nickel at normal incidence. The standard deviation of the range distribution, known as straggling, is $\sim 0.2 \mu \mathrm{m}$ at $3.5 \mathrm{MeV}$. The relative magnitude of the distribution peaks decrease with increasing energy as the straggling increases, keeping the area under each curve constant. Discarding the first layer, to minimize tritium contamination, results in a minimum detection energy of $\sim 0.5 \mathrm{MeV}$.

Fig. 8(b) shows the TRIM calculated range distributions for $\mathrm{He}$ ions at $3.5 \mathrm{MeV}$ into nickel at various angles of incidence. The widths of the range distributions increase with incident angle because transverse straggling is more significant than longitudinal straggling. These distributions are combined with the predicted incident angle distributions to obtain the expected range distribution for first orbit loss in each nickel foil stack.

The foils are actually curved since they are wrapped around a cylindrical spool inside the probe head. This curvature can cause particles to strike the foil at larger incident angles than if the foil were flat, resulting in a more shallow implantation ranges. However, this curvature can only be responsible for spreading of the first orbit loss range distribution to, at most, one foil layer shallower. It has little effect on the position of the peak in the range distribution. This effect is neglected along with other spreading effects such as nonuniformities in foil thickness, which may be responsible for the spreading of calibration sample results seen in Ref [10], and Doppler broadening of the birth energy distribution. These effects taken together can be expected to spread the depth distributions of first orbit loss by one foil layer in either direction.

In the next section, the first orbit loss model predictions generated through the use of ORBIT, PORT and TRIM are compared to alpha collector measurements of DT plasmas.

\section{EXPERIMENTAL RESULTS}

In each part of this section, a characteristic of the observed loss is compared to the first orbit loss model first for the two exposures conducted at a plasma current of 1.0 MA (exposures $C \& E$ ), then for the two exposures conducted at 1.8 MA (exposures $B$ \& D). A comparison is then made with the lost alpha scintillator images for each exposure.

Exposures B through D are nominally identical discharges with the exception of the two values of plasma current and slight modifications that can be seen in Table I. Note that exposures B \& C used the original probe head, and exposures D \& E used the redesigned head with collimating ports that were twice as deep, and the head rotated clockwise $7.5^{\circ}$ (see section 2.1.3).

When a fluence measurement is compared to the first orbit model only the summed fluence of foil layers 4 through 9 are included. Due to the geometry of the collimating ports and the predicted range distribution of $3.5 \mathrm{MeV}$ alphas (see Fig. 8), it is to be expected that essen- 

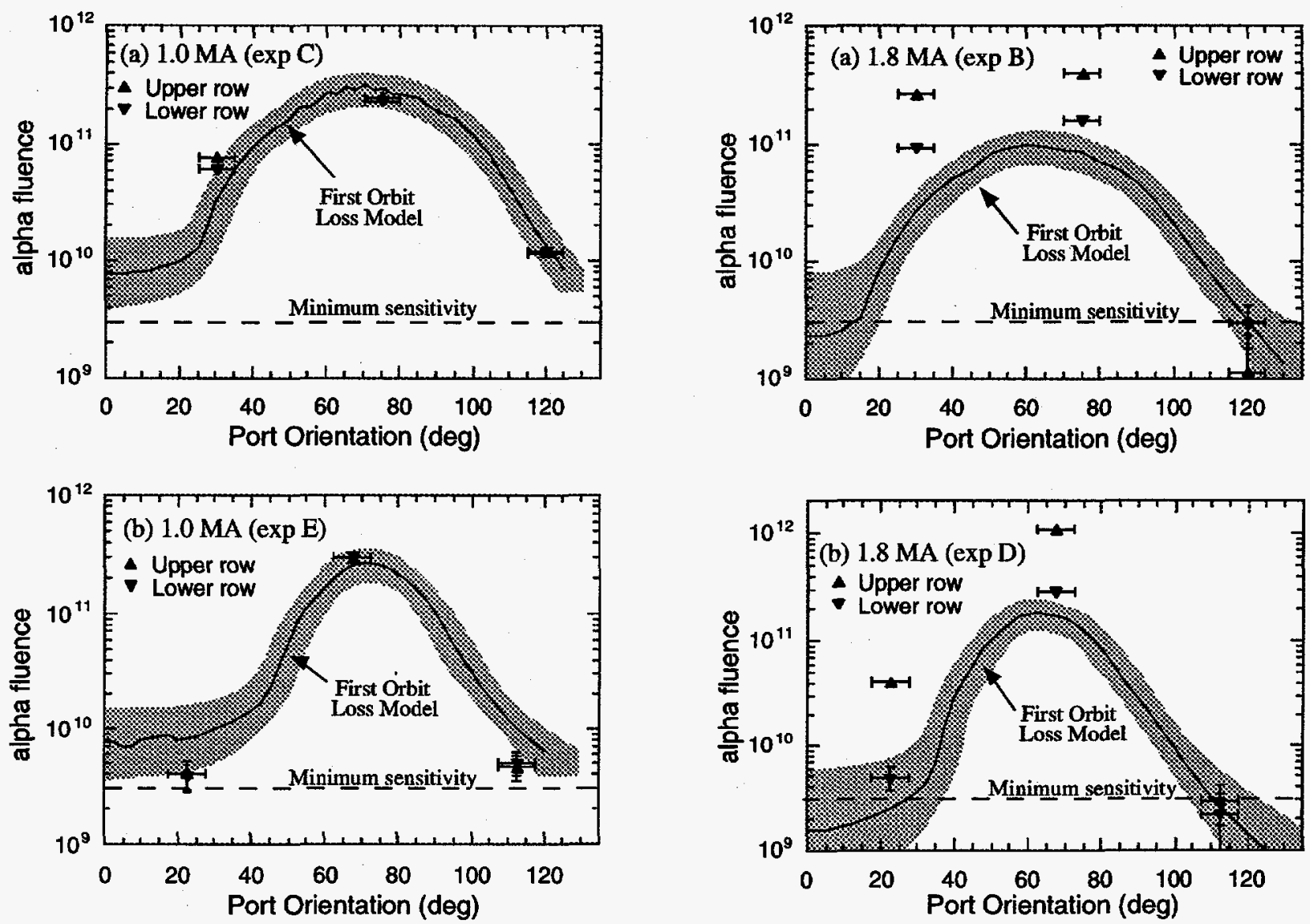

FIG. 9. $I_{p}=1.0 \mathrm{MA}$ - Measured fluences of He in layers 4 through 9 for the upper and lower rows of $(a)$ exposure $C$ using the original probe head design, and (b) exposure $E$ using the redesigned probe head, compared with the first orbit loss model calculated with the Lorentz ORBIT code as a function of port orientation. The dashed line, representing the minimum sensitivity, is approximated by multiplying the minimum sensitivity per layer $\left(5 \times 10^{8}\right.$ alphas $)$ by 6 layers. The vertical error for data points above $1.2 \times 10^{10}$ alphas is $\sim \pm 10 \%$, corresponding approximately to the height of the triangular data symbol.

tially no first orbit lost alpha will be stopped by foil layers shallower than $3 \mu \mathrm{m}$, nor penetrate deeper than $9 \mu \mathrm{m}$. Layers 1 through 3 , and 10 were therefore excluded from comparisons against first orbit loss since $\mathrm{He}$ in these layers must be due to other sources such as thermalized alpha ash or externally introduced He puffs.

Analysis of foils from the inboard facing ports (towards the center of the torus) resulted in levels of implanted He below or near the minimum sensitivity ( $\sim 5 \times 10^{8}$ alphas). This was as expected for the reasons discussed in section 3.1. Therefore, only the outboard facing ports are compared to the first orbit loss model in this section. Although there was essentially no He implanted in these inboard facing samples, it was important to obtain these results to verify expectations and to provide addi-

FIG. 10. $I_{p}=1.8 \mathrm{MA}-$ Measured fluences of He in layers 4 through 9 for the upper and lower rows of, (a) exposure $B$ using the original probe head design, and (b) exposure $D$ using the redesigned probe head, compared with the first orbit loss model. Same notation as Fig. 9. The $30^{\circ}$ and $75^{\circ}$ ports reveal the existence of an anomalous loss at 1.8 MA.

tional insight into any anomalous results that may be obtained.

\subsection{Absolute Fluence}

\subsection{1. $I_{p}=1.0$ MA Absolute Fluence}

Fig. 9 shows the comparison between the observed fluence (in number of alphas collected) to the upper and lower rows of outboard facing ports $\left(30^{\circ}, 75^{\circ}, 120^{\circ}\right)$ at 1.0 MA (exposures $\mathrm{C} \& \mathrm{E}$ ), along with the first orbit loss model predictions generated with the Lorentz ORBIT code (see section 3) for the upper row as a function of collimating port orientation. The first orbit loss model for the lower row is not plotted because it is essentially unchanged from the model for the upper row. The first orbit loss model curve is the predicted alpha collection fraction (as seen in Fig. 6 for 1.8 MA exposures) times the total neutron production for each shot (see section 3 ). 
The observed fluence is plotted for the total He content of layers 4 through 9 . The observed fluence agrees very well with the first orbit loss model, within the uncertainties discussed in section 4.5.

\subsection{2. $I_{p}=1.8$ MA Absolute Fluence}

Fig. 10 (analogous to Fig. 9) shows the comparison between the observed fluence and the first orbit loss model at 1.8 MA (exposures B \& D). The results of the $30^{\circ}$ and $75^{\circ}$ ports indicate that an anomalously large loss exists, with an alpha fluence roughly an order of magnitude larger than is expected for first orbit loss to the upper rows. This anomalous loss is significantly diminished in the lower rows. The $120^{\circ}$ ports, however, appear to be in agreement (keeping in mind that they are near minimum sensitivity) with the first orbit loss model, within the uncertainties discussed in section 4.5. This indicates that the anomalous loss does not extend to pitch angles as large as does first orbit loss.

The samples with the largest fluence for each of the 1.8 MA exposures, corresponding to the upper $75^{\circ}$ port for exposure $B$ and the upper $67.5^{\circ}$ port for exposure $D$, show an increase by a factor of 4.7 and 6.4 respectively when the measured fluence in layers 4 through 9 is compared to the predicted fluence for first orbit loss. When the total measured fluence of layers 2 through 10 is compared to the predicted fluence, both of these samples show an increase by a factor of $\sim 7$. Assuming that the predicted first orbit loss is included in these measurements makes the anomalous loss $\sim 6$ times larger than first orbit loss.

\subsubsection{Comparison with Lost Alpha detector}

Fig. 11 shows the dependence of the $90^{\circ}$ scintillator signal on plasma current for shots $B$ through $E$. The data is normalized to the model at 1.0 MA due to uncertainties in the absolute calibration [1]. The gray area overlaid on this plot is taken from the analysis of Ref. [1]. It represents the first orbit loss model with uncertainties as calculated for $R=2.52 \mathrm{~m}$ plasmas, but appears to fit the model predictions at $R=2.45 \mathrm{~m}$ quite well. The magnitude of alpha loss as measured with the $90^{\circ}$ scintillator agrees with the first orbit loss model within the uncertainties. There is no increase in the signal at 1.8 MA with respect to the model, consistent with the behavior observed in all other plasma discharges. The first orbit loss model predicts a drop in the alpha collection fraction by a factor of $\sim 3.4$ between 1.0 MA and 1.8 MA. An anomalous loss causing a factor of 7 increase at 1.8 MA would make the 1.8 MA alpha collection fraction a factor of $\sim 2$ larger than at 1.0 MA. Hence, it appears that the anomalous loss

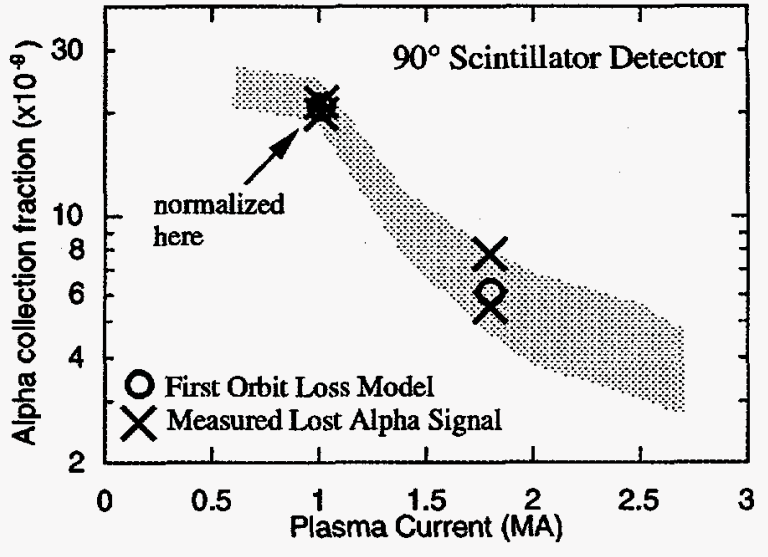

FIG. 11. Plasma current dependence of the alpha collection fraction measured with the $90^{\circ}$ lost alpha scintillator detector normalized to the first orbit loss model at 1.0 MA. The shaded region corresponds to the first orbit loss model calculated for $R=2.52 \mathrm{~m}$ plasmas in Ref. [1], but appears to fit the $R=2.45 \mathrm{~m}$ data well. The good agreement with the model implies that this detector does not 'see' the anomalous loss apparent at $1.8 \mathrm{MA}$ using the alpha collector probe.

detected at 1.8 MA with the alpha collector is not seen by the $90^{\circ}$ lost alpha scintillator detector.

\subsection{Energy Distribution}

\subsection{1. $I_{p}=1.0 \mathrm{MA}$ Energy Distribution}

Fig. 12 shows the comparison between the measured range distribution and the first orbit loss model of 3.5 MeV alphas generated with the ORBIT, PORT, and TRIM codes (see section 3 ) for the upper and lower row of outboard facing ports at 1.0 MA (exposures $\mathrm{C} \& \mathrm{E}$ ). There is reasonable agreement between the observed distribution and the model, with the exception of a low energy loss feature appearing in layers 2 and 3 corresponding to an energy below $2.0 \mathrm{MeV}$. Although the shape of the peaks lying between layers 4 and 9 do not exactly match the first orbit loss model peaks, the important feature is that the peaks appear in approximately the same layers at an overall magnitude determined in section 4.1.1 to be in agreement with first orbit loss. The disagreement in the specific shape of the distributions may be attributable to the neglect of foil nonuniformities, curvature of the foils, and Doppler broadening of the birth energy distribution (see section 3). Overall, the agreement is sufficient to conclude that the observations are consistent with the model for first orbit loss with the exception of a low energy loss feature that is not yet understood, but might be related to the anomalous loss at 1.8 MA. 

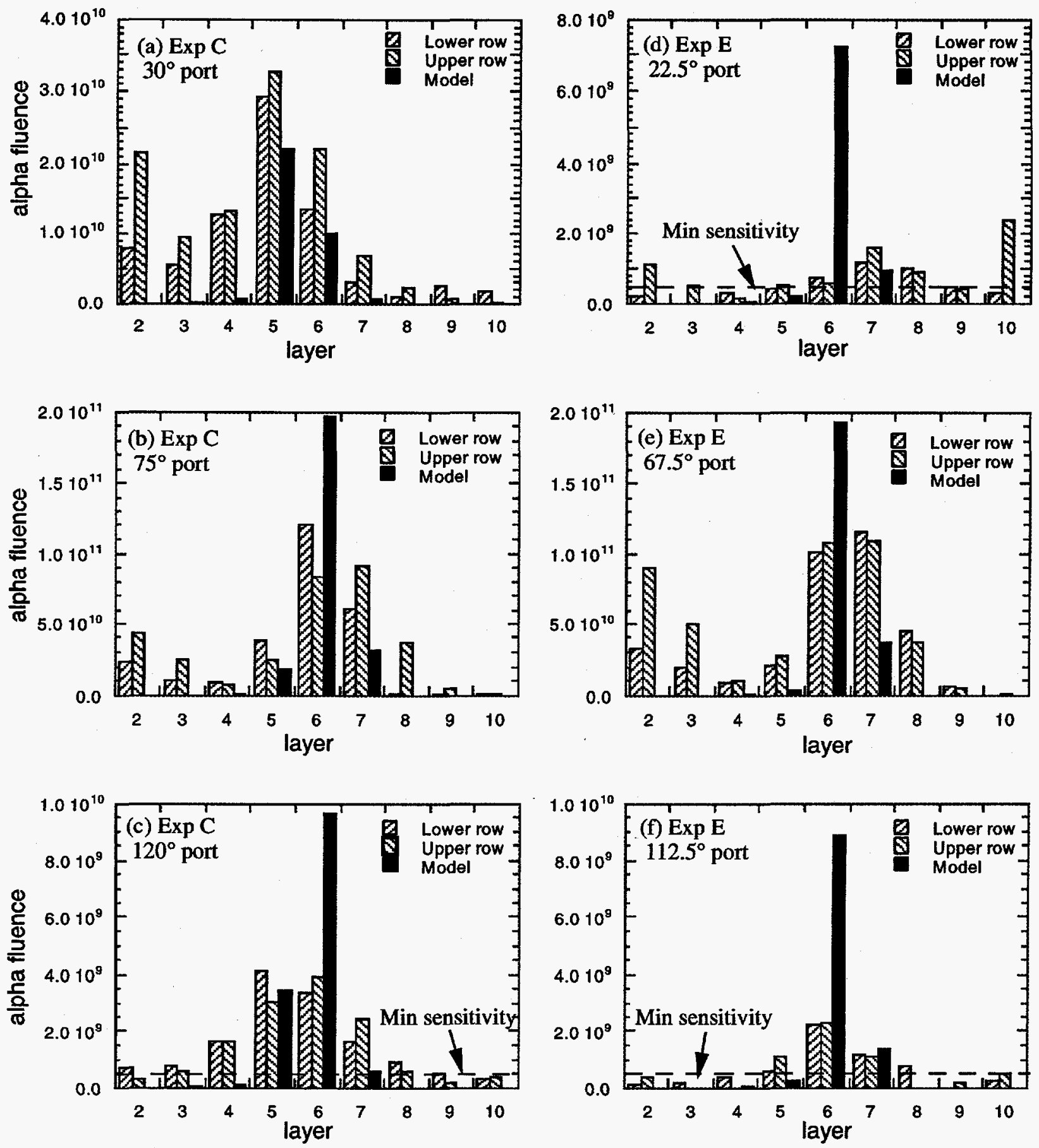

FIG. 12. $I_{p}=1.0 \mathrm{MA}$ - Implantation range distributions as a function of layer (ie. 1 um bins) for the upper and lower rows and model predictions for $(a) 30^{\circ}$ (b) $75^{\circ}$ and (c) $120^{\circ}$ ports of exposure $C$, and (d) $22.5^{\circ}$ (e) $67.5^{\circ}$ and $(f) 112.5^{\circ}$ ports of exposure E. Notice that the vertical scales are linear and not all the same. The plots with a maximum of the vertical scale $\leq 2 \times 10^{10}$ alphas have the minimum sensitivity of the sample analysis ( $5 \times 10^{8}$ alphas/layer) represented by a dashed line.

\subsection{2. $I_{p}=1.8$ MA Energy Distribution}

Fig. 13 (analogous to Fig. 12) shows the comparison between the measured range distribution and the first orbit loss model for the upper and lower rows of outboard fac- ing ports at 1.8 MA (exposures B \& D). The observed peak in the range.distribution occurs at a shallower depth and has a significantly larger width than the first orbit loss model for the $30^{\circ}$ and $75^{\circ}$ ports of exposure $B$, and the $67.5^{\circ}$ port of exposure D (Figs. 13(a,b,e)). This indicates 

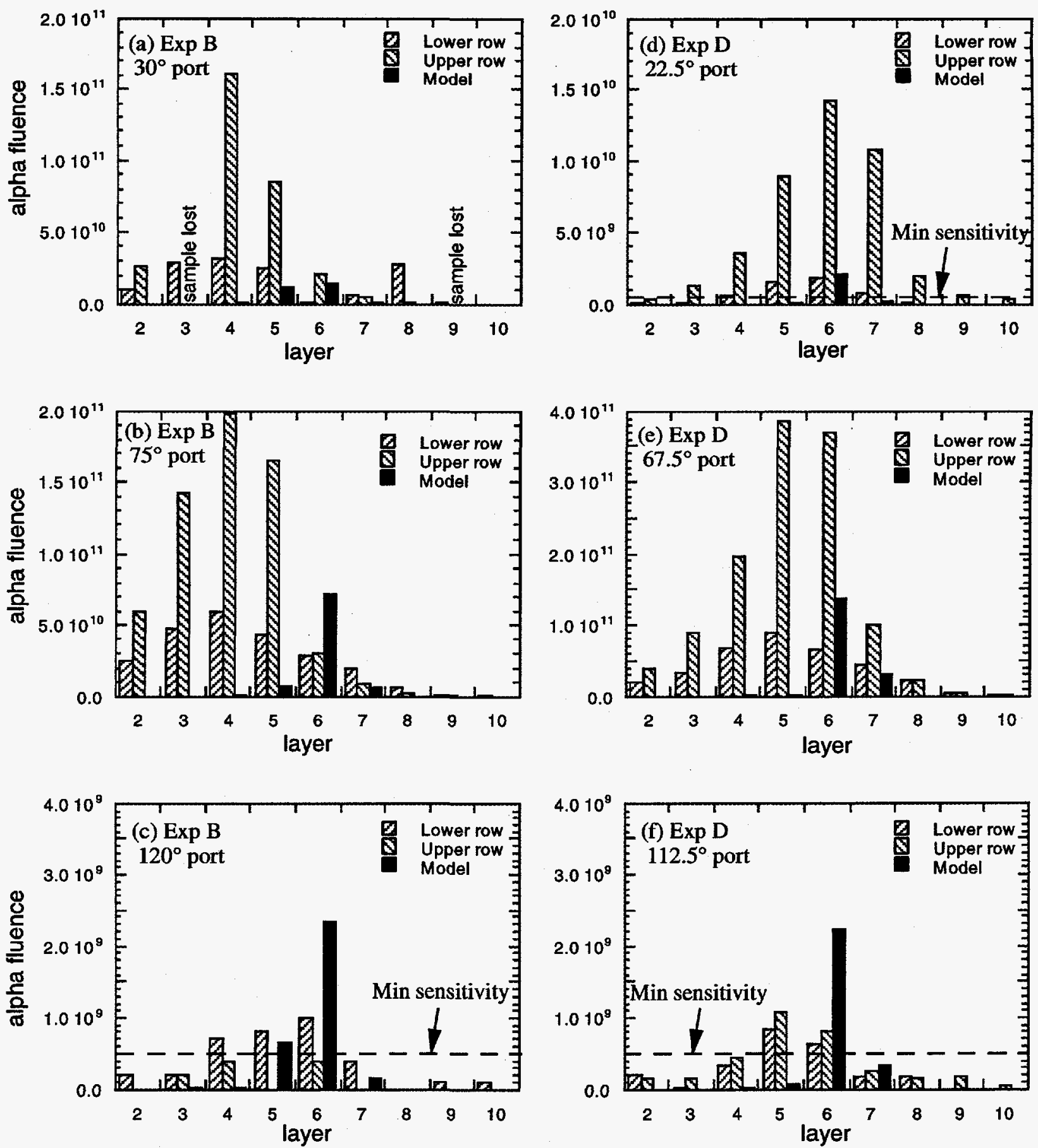

FIG. 13. $I_{p}=1.8 \mathrm{MA}$ - Implantation range distributions as a function of layer for the upper and lower rows and model predictions for (a) $30^{\circ}$ (b) $75^{\circ}$ and (c) $120^{\circ}$ ports of exposure B, and (d) $22.5^{\circ}$ (e) $67.5^{\circ}$ and $(f) 112.5^{\circ}$ ports of exposure D. Same notation as Fig. 12. Layers 3 and 9 of the upper row of the $30^{\circ}$ port of exposure $B$ in (a) were lost during the tritium decontamination process [9].

that the anomalous loss occurring at 1.8 MA consists of partially thermalized alphas.

The data from the $22.5^{\circ}$ ports (upper and lower rows) from exposure D (Fig. 13(d)) seem to indicate an alpha loss near the birth energy and not the partially thermalized loss mentioned above. This is an indication that the partially thermalized anomalous loss does not occur at pitch angles below the maximum pitch angle accepted by this port $\left(\sim 49^{\circ}\right)$. Thus, the observed signal should correspond to purely first orbit loss of passing particles. However, as seen in Fig. 10(b), the total fluence for layers 49 of the upper $22.5^{\circ}$ port was nearly 17 times expected 
first orbit loss. The peak in the sixth layer is repeated in the lower row of the $22.5^{\circ}$ port, as seen in Fig. 13(d), verifying the lack of a partially thermalized loss to this location. Also, the total fluence for the lower row seen in Fig. 10(b) is consistent with first orbit loss. Although there does appear to be an anomalously large signal to the upper row, until this result can be shown to be reproducible, it will be assumed that the loss to this port is purely first orbit loss and is not associated with the partially thermalized anomalous loss observed at 1.8 MA. If this is a valid result, it is associated with a prompt loss mechanism, not the delayed mechanism (allowing for time to slow down) responsible for the partially thermalized anomalous loss.

The peak in the fourth layer of the $75^{\circ}$ port of exposure B (Fig. 13(b)) corresponds to an alpha loss energy of $\sim 2.2 \pm 0.3 \mathrm{MeV}$ (see Fig. 8) if normal incidence is assumed. Similarly, the peak in the fifth layer of the $67.5^{\circ}$ port of exposure D (Fig. 13(d)) corresponds to an alpha loss energy of $\sim 2.7 \pm 0.3 \mathrm{MeV}$. Exposure D used the redesigned probe head with the improved collimation (ie. particles implant closer to normal incidence), and thus provides a better indication of alpha loss energy than does exposure $\mathrm{B}$, which used the original design. It might also be inferred from the deeper implantation range in exposure $\mathrm{D}$ that the $7.5^{\circ}$ clockwise rotation that went into the redesign was such as to bring the axis of the port closer to the pitch angle of the anomalous loss, also allowing particles to implant closer to normal incidence. In other words, the anomalous loss probably occurs at a pitch angle closer to $67.5^{\circ}$ than to $75^{\circ}$. Assuming the two $1.8 \mathrm{MA}$ exposures are exposed to an anomalous loss of the same energy, the peak of the energy distribution is inferred to be $\sim 2.5 \pm$ $0.3 \mathrm{MeV}$ (ie. $\sim 70 \pm 10 \%$ of the birth energy). In the discussion of section 5 , the anomalous loss at 1.8 MA will be simplified as having a single loss energy of $2.5 \mathrm{MeV}$.

\subsubsection{Comparison with Lost Alpha detector}

The gyroradius distributions for exposures $B$ through $\mathrm{E}$ as measured with the $90^{\circ}$ lost alpha scintillator detector are shown in Fig. 14. Two model curves, taking into account the finite aperture sizes and optical resolutions of the detectors, are also plotted. The distributions show good shot to shot consistency, independent of the plasma current, and they agree closely with the model assuming alpha loss at a single energy of $3.5 \mathrm{MeV}$. For comparison with what might be expected if some of the anomalous loss observed with the alpha collector were also detected by the $90^{\circ}$ scintillator detector, the other model assumes equal loss components at energies of $2.5 \mathrm{MeV}$ and $3.5 \mathrm{MeV}$. These distributions provide further evidence that the lost alpha scintillator detectors do not detect the

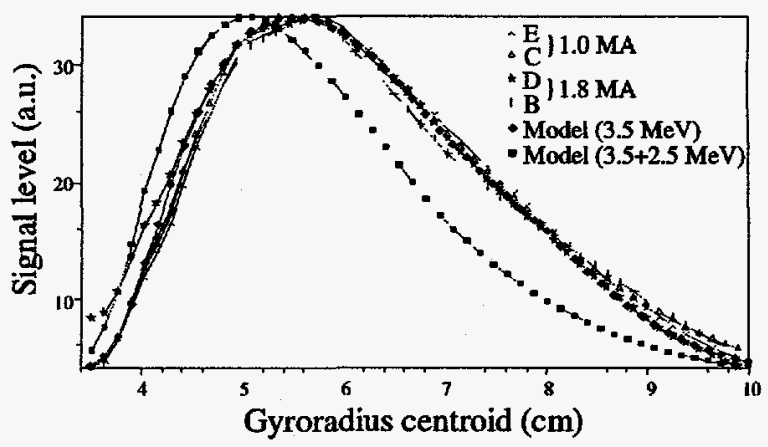

FIG. 14. Gyroradius distributions of alpha loss as measured with the $90^{\circ}$ lost alpha scintillator detector averaged over toroidal pitch angle, from $45^{\circ}$ to $90^{\circ}$, and time, from 3.4 to $3.7 \mathrm{sec}$ (ie. the quasi-steady state portion of the discharge). Model curves are plotted for $3.5 \mathrm{MeV}$ alphas and equal fluxes of $2.5 \mathrm{MeV}$ and $3.5 \mathrm{MeV}$ alphas after being correctedfor the finite aperture sizes and optical resolutions of the detectors. The curves are normalized vertically to each other near their peaks, but the horizontal axes were absolutely calibrated by an in-vessel alignment to within $\sim 1 \mathrm{~cm}$.

partially thermalized anomalous loss observed with the alpha collector at 1.8 MA, which was inferred to be up to 6 times the first orbit loss at an energy of $\sim 2.5 \mathrm{MeV}$.

\subsection{Pitch Angle Distribution}

\subsection{1. $I_{p}=1.0$ MA Pitch Angle Distribution}

The first orbit model predictions in Fig. 9 and 10 are just the expected pitch angle distributions corrected for the geometric resolution of the detector (see section 3). So the pitch angle distributions of Fig. 9 for the exposures at a plasma current of 1.0 MA (C \& E) show good agreement between the observations and the first orbit loss model. However, the wide pitch angle acceptance of the collimating ports $\left( \pm 45^{\circ}\right.$ for the original design; $\pm 27^{\circ}$ for the redesign) result in relatively poor pitch angle resolution. To obtain improved information pertaining to the pitch angle distribution, it was decided to cut selected samples vertically in half to compare the fluences contained in the right and left halves. A loss at a pitch angle larger than the collimating port's orientation tends to concentrate $\mathrm{He}$ in the left half of the foil stack, assuming $r \gg w, d$ and $\chi_{0}>0$ (see sections 2.1 and 3 for symbol definitions).

The PORT code was used to predict the fraction of alpha fluence implanted in the left half of the foil stack, referred to as the left collection fraction, for the pitch angle distribution generated for first orbit loss using the Lorentz ORBIT code. In other words, the left collection fraction $L$ is:

$L=\frac{l}{l+r}$ 

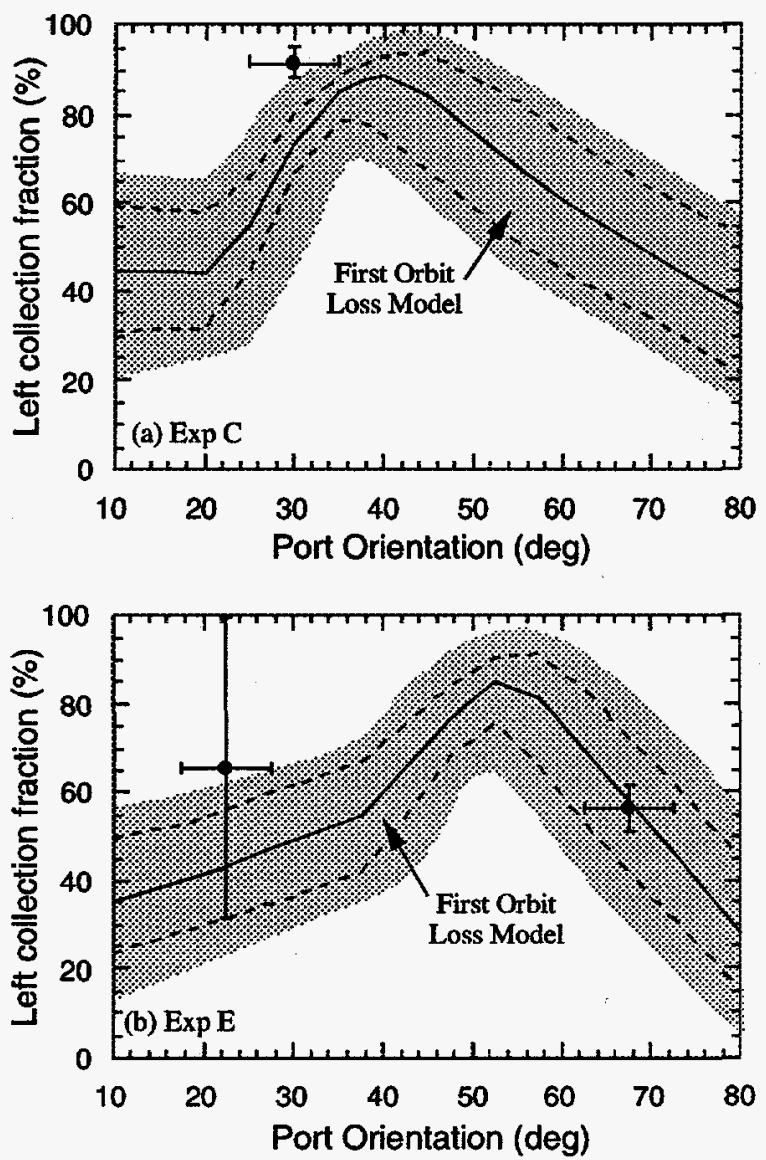

FIG. 15. $I_{p}=1.0 \mathrm{MA}$ - Left collection fraction as a function of port orientation for the upper row of the (a) $30^{\circ}$ port of exposure $C$, and (b) $22.5^{\circ}$ and $67.5^{\circ}$ ports of exposure $E$. The left collection fraction is the fraction of He in layers 4 through 9 that implanted in the left half of the stack. The model is based on the first orbit loss pitch angle distributions calculated using ORBIT. The dashed lines represent the model assuming the cut is made $10 \%$ of the port width to the right (upper dashed line) or left (lower dashed line) of center.

where $l$ and $r$ are the amounts of He contained in the left and right halves respectively. Fig. 15 shows a comparison between the fraction of He detected in the left half for the selected samples at 1.0 MA and the model prediction as a function of port orientation. The solid line represents the expected left collection fraction assuming the cut was made right down the middle of the foils. The dashed lines represent the expected left collection fractions for the cases where the cut is made just $10 \%$ to the left or right of center of the port diameter (ie. $\sim 0.6 \mathrm{~mm}$ ). Notice that the model appears to slightly underestimate the left collection fraction at the $30^{\circ}$ port. A possible explanation for this will be discussed in section 4.5. Otherwise, there is reasonable agreement between the first orbit loss model and the data.
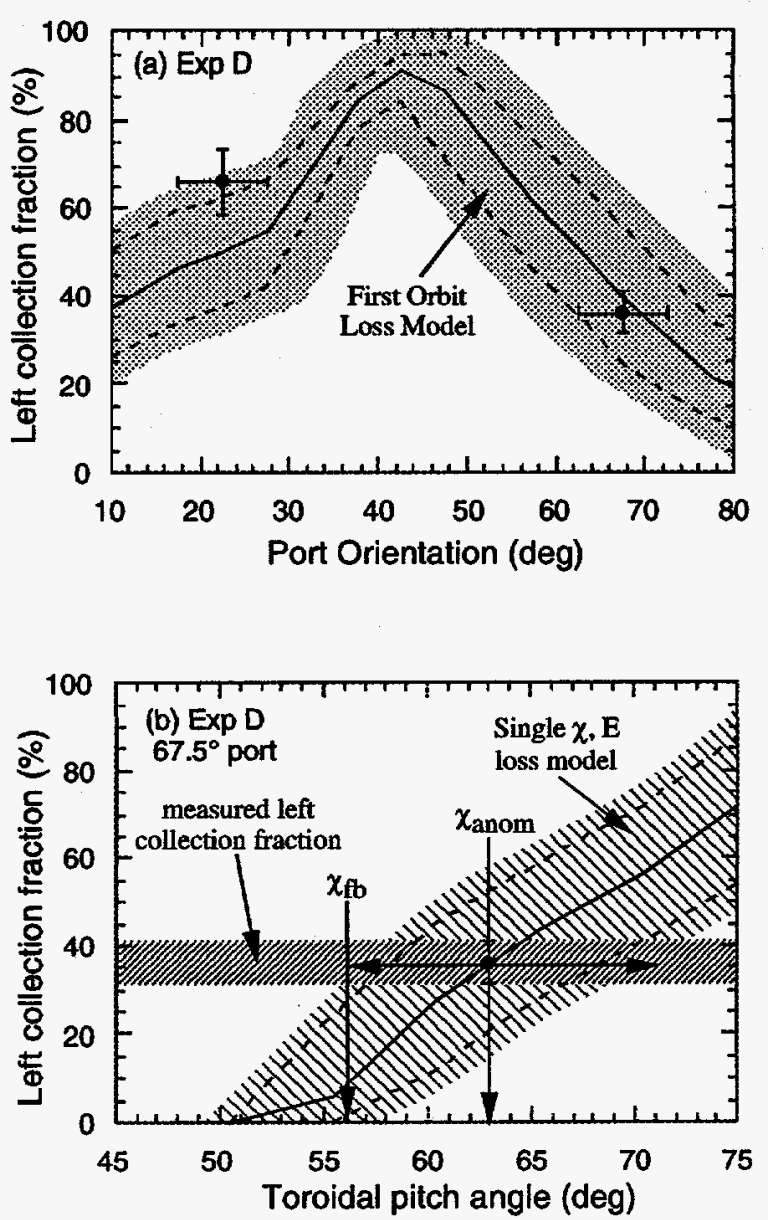

FIG. 16. $I_{p}=1.8 \mathrm{MA}-$ (a) Left collection fraction as a function of port orientation for the upper row of the $22.5^{\circ}$ and $67.5^{\circ}$ ports of exposure D, analogous to Fig. 15. (b) Left collection fraction for the upper $67.5^{\circ}$ port of exposure $D$ fit to a model based on alpha loss at a single energy (2.5 MeV) and a single toroidal pitch angle as a function of this pitch angle. From the data, a toroidal pitch angle for the anomalous loss of $63^{\circ} \pm 7^{\circ}$ is inferred.

\subsection{2. $I_{p}=1.8$ MA Pitch Angle Distribution}

Fig. 16(a) (analogous to Fig. 15) shows the comparison between the fraction of $\mathrm{He}$ detected in the left half of the foil stacks for the $22.5^{\circ}$ and $67.5^{\circ}$ ports of exposure $\mathrm{D}$ and the model prediction as a function of port orientation. Again, notice that the model appears to underestimate the left collection fraction at the $22.5^{\circ}$ port. The observation at $67.5^{\circ}$ is in good agreement with the model for $3.5 \mathrm{MeV}$ alphas lost with the pitch angle distribution calculated using the Lorentz ORBIT code. However, the majority of the loss detected by this port is the partially thermalized anomalous loss.

The PORT code can be used to find the toroidal pitch angle for the anomalous loss that produces the best match 


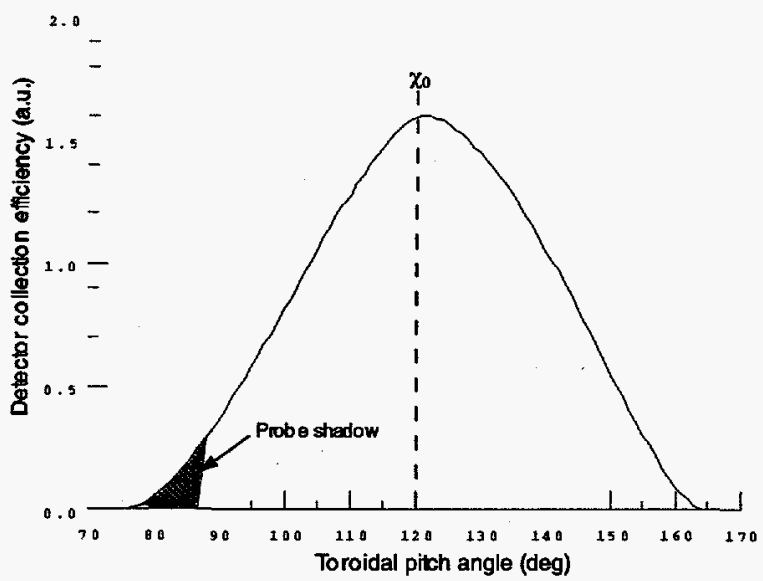

FIG. 17. $I_{p}=1.8 \mathrm{MA}$ - Relative detector collection efficiency as a function of toroidal pitch angle for the $120^{\circ}$ port of the original probe head design (exposure B). The collection efficiency drops off away from the pitch angle of the port axis $\left(\chi_{0}\right)$ due to collimation, and is cut off at $\pm 45^{\circ}$ either side of $\chi_{0}$. The probe shadow occurs near a true pitch angle of $\chi=90^{\circ}$ $\left(\chi_{t}=\chi-\chi_{b t} \approx 85^{\circ}\right)$ where the gyro-orbit of a high energy ion intercepts the probe head.

to the observed left collection fraction. This assumes that the anomalous loss can be represented by a loss at a single pitch angle and single energy of $2.5 \mathrm{MeV}$. Fig. 16(b) shows that the measured left collection fraction, at $\sim 36 \% \pm 5 \%$, best matches the model for $2.5 \mathrm{MeV}$ alphas at a toroidal pitch angle of $\sim 63^{\circ} \pm 7^{\circ}$. The toroidal pitch angle of the fattest banana orbit $\chi_{f b}$ which corresponds to the boundary between passing and trapped particles was seen in Fig. 1 to oecur at $\chi_{f b} \approx 56^{\circ}$ for $I_{p}=1.8 \mathrm{MA}$. Thus it is concluded that $\chi_{a n o m}>\chi_{f b}$ under the preceding assumptions.

The extent of the anomalous loss in pitch angle can be further narrowed down by its absence in some of the detector samples. The pitch angle distributions of Fig. 10 for the exposures at a plasma current of 1.8 MA (B \& D) show that there is no anomalous loss being detected by the $120^{\circ}\left(112.5^{\circ}\right)$ port of exposure B (D), indicating that the anomalous loss does not extend to pitch angles as large as first orbit loss as previously mentioned in section 4.1.2. Fig. 17 shows the relative detector collection efficiency (ie. the fraction of particles that reach the foil without being stopped by collimation), assuming flat pitch and gyro angle distributions, as a function of the toroidal pitch angle, $\chi_{t}=\chi-\chi_{b t}$ (see section 3 ), for the $120^{\circ}$ port of exposure $\mathrm{B}$ for alphas at $2.5 \mathrm{MeV}$ (the representative anomalous loss energy). The pitch angle acceptance of $\pm 45^{\circ}$ for the original probe design, centered approximately about the port's orientation of $120^{\circ}$ for exposure B can be seen in this figure. Incidentally, the data from this port represents the first experimental measurement of par- ticles escaping to the TFTR wall on counter-going orbits (ie. $\chi>90^{\circ}$ ). Although the signals are small in the $120^{\circ}$ ports, as evidenced in Figs. 9, 10, 12, and 13, the results appear to be consistent with first orbit loss.

Also shown in 17 is the self-shadowing effect of the probe head. This shadowing is the result of insufficient displacement of an alpha along the magnetic field line making the alpha unable to clear the probe head in one gyro-orbit when the true pitch angle, $\chi$, is near $90^{\circ}$ $\left(\chi_{b t}=4.6^{\circ}\right.$ for a $1.8 \mathrm{MA}$ exposure explains the shift away from $\chi_{t}=90^{\circ}$ in 17). So, with the exception of a small degree of acceptance at $75^{\circ}$ to $80^{\circ}$ for exposure $B$, the effective minimum toroidal pitch angle cutoff occurs at $\chi_{t}>87^{\circ}$. The use of the redesigned probe with its smaller pitch angle acceptance range in exposure $D$ is slightly more restrictive. This sets the upper limit on the toroidal pitch angle of the anomalous loss at $87^{\circ}$.

Similarly, the toroidal pitch angle acceptance for the $30^{\circ}\left(22.5^{\circ}\right)$ port extends up to $\chi_{t}=75^{\circ}\left(49.1^{\circ}\right)$ for exposure $B$ (D). Probe shadowing is not a factor in these ports since pitch angles near $90^{\circ}$ are excluded. It was seen in section 4.2.2 that the partially thermalized anomalous loss was detected in the $30^{\circ}$ port of exposure $B$, but not in the $22.5^{\circ}$ port of exposure $D$ (although the anomalously large alpha fluence in this port is not fully understood). Thus the anomalous loss occurs at toroidal pitch angles $\chi_{t}<75^{\circ}$, but not at $\chi_{t}<49^{\circ}$. This sets the lower limit on the toroidal pitch angle of the anomalous loss, $\chi_{\text {anom }}$, at $49^{\circ}$. Combining these results yields, $49^{\circ} \leq \chi_{\text {anom }} \leq 87^{\circ}$. It might, however, be expected that a feasible anomalous loss mechanism would preferentially provide either trapped or passing particles. Since the best fit in Fig. 16(b) gave $\chi_{a n o m}>\chi_{f b}$, trapped particles are probably the main constituent of the anomalous loss. It thus seems likely that the anomalous loss pitch angle distribution is concentrated in a narrow range above the passing-trapped boundary (ie. $56^{\circ}<\chi_{\text {anom }}<87^{\circ}$ ).

\subsubsection{Comparison with Lost Alpha detector}

The toroidal pitch angle distributions measured with the $90^{\circ}$ lost alpha scintillator detector are shown in Fig. 18. For comparison, the Lorentz ORBIT generated pitch angle distributions are plotted after being corrected for the geometric and optical resolutions of the detectors. Notice that the model at $I_{p}=1.0 \mathrm{MA}$ in Fig. 18(a) tends to overestimate the toroidal pitch angle of first orbit loss (ie. the model is shifted to the right of the measured distribution) by $\sim 6^{\circ}$. This discrepancy will be referred to in section 4.5. The peaks of the $I_{p}=1.8 \mathrm{MA}$ distributions in Fig. 18(b), however, agree to within the $3^{\circ}$ uncertainty associated with the scintillator detectors. The shapes of 

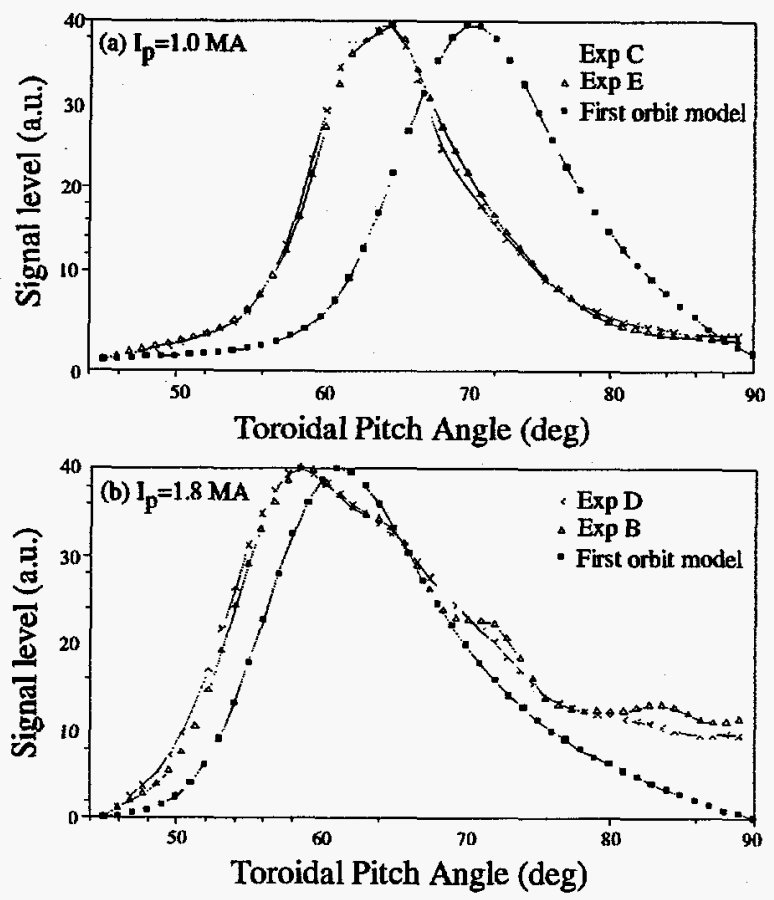

FIG. 18. Toroidal pitch angle distributions of alpha loss as measured with the $90^{\circ}$ lost alpha scintillator detector averaged over gyroradius, from 3.5 to $9.9 \mathrm{~cm}$, and time, from 3.4 to $3.7 \mathrm{sec}$. Model curves are plotted for the ORBTT calculated first orbit loss after being corrected for the finite aperture sizes and optical resolutions of the detectors. The curves are normalized vertically to each other near their peaks, but the horizontal axes were absolutely calibrated by an in-vessel alignment to within $\sim 3^{\circ}$

the model distributions are in reasonable agreement with the measured ones. The distribution at 1.8 MA appears to be somewhat wider than predicted, but there is no indication of an anomalously large loss occurring at a pitch angle above the fattest banana orbit.

\subsection{Radial Distribution}

\subsection{1. $I_{p}=1.0$ MA Radial Distribution}

It is apparent from Fig. 9 that the fluences of alphas at a plasma current of $1.0 \mathrm{MA}$ to the upper and lower rows of outboard ports are comparable. Fig. 19 shows the fluence levels for the 1.0 MA exposures (C \& E) measured in layers 4-9 (representing first orbit loss) of the $75^{\circ}$ upper and lower ports and compares them to the first orbit loss model as a function of detector height as measured from the midplane. The modeled fluence drops sharply about $1.0 \mathrm{~cm}$ outside the RF limiter radius due to the shadowing effect of the limiter. Although the lower row of ports was placed below the RF limiter, the outward bulge of the magnetic field (by $\sim 1.5 \mathrm{~mm} \mathrm{[16])} \mathrm{between} \mathrm{TF} \mathrm{coils} \mathrm{asso-}$ ciated with TF ripple, and the downward drifts (grad B and curvature) that an alpha experiences during its transit from the limiter to the probe is sufficient to keep the lower ports out of the limiter shadow.

\subsection{2. $I_{p}=1.8$ MA Radial Distribution}

Fig. 10 showed that the fluences of alphas at a plasma current of $1.8 \mathrm{MA}$ for the $30^{\circ}$ and $75^{\circ}$ ports drops by a factor of 3 or more between the upper and lower rows. Fig. 20 (analogous to Fig. 19) shows the fluence levels for the 1.8 MA exposures (B \& D) of the $75^{\circ}$ upper and lower ports and compares them to the first orbit loss model as a function of detector height. A strong radial dependence is clearly present among the 1.8 MA anomalous loss data. It is unclear whether this radial dependence is due to the RF limiter shadowing or a radial diffusive loss of alphas. The shadowing effect of the limiter increases when the alpha orbits stay closer to the magnetic field lines as occurs with decreased alpha energy, decreased alpha pitch angle, or increased plasma current. Lorentz ORBIT code simulations show that for a $2.5 \mathrm{MeV}$ alpha, the limiter shadow is brought only $\sim 1 \mathrm{~mm}$ closer to the RF limiter radius than is shown in Fig. 20 for first orbit loss, making it unlikely that the strong radial dependence is due solely to limiter shadowing. A diffusive loss having a finite random radial step-size between toroidal transits, could give rise to a radial dependence near absorbing boundaries such as the $R F$ limiter and the probe head itself (see section 5.3). Further experiments that vary the radial position of the probe would be necessary to obtain a conclusive result. However, the strong radial dependence is further evidence that the 1.8 MA loss is not pure first orbit loss.

\subsubsection{Comparison with Lost Alpha detector}

The $90^{\circ}$ scintillator detector with which the alpha collector results are compared is fixed in position such that a radial scan is not possible. As pointed out in section 2.1.2, the pinhole aperture of the scintillator detector is located $\sim 0.9 \mathrm{~cm}$ lower than the top of the lower row of the alpha collector. Therefore, the strong radial dependence of the anomalous loss might explain why it isn't observed on the $90^{\circ}$ scintillator.

The scintillator detector located $20^{\circ}$ below the outer midplane is, however, moveable. Radial scans have been accomplished using this probe to investigate the diffusive nature of stochastic ripple diffusion (SRD) of fusion products. SRD causes alphas to be lost near their birth energy and can be the dominant loss mechanism in a narrow poloidal region about the outer midplane. These scans have shown a radial dependence of alpha loss comparable to that of the 1.8 MA anomalous loss observed using the 

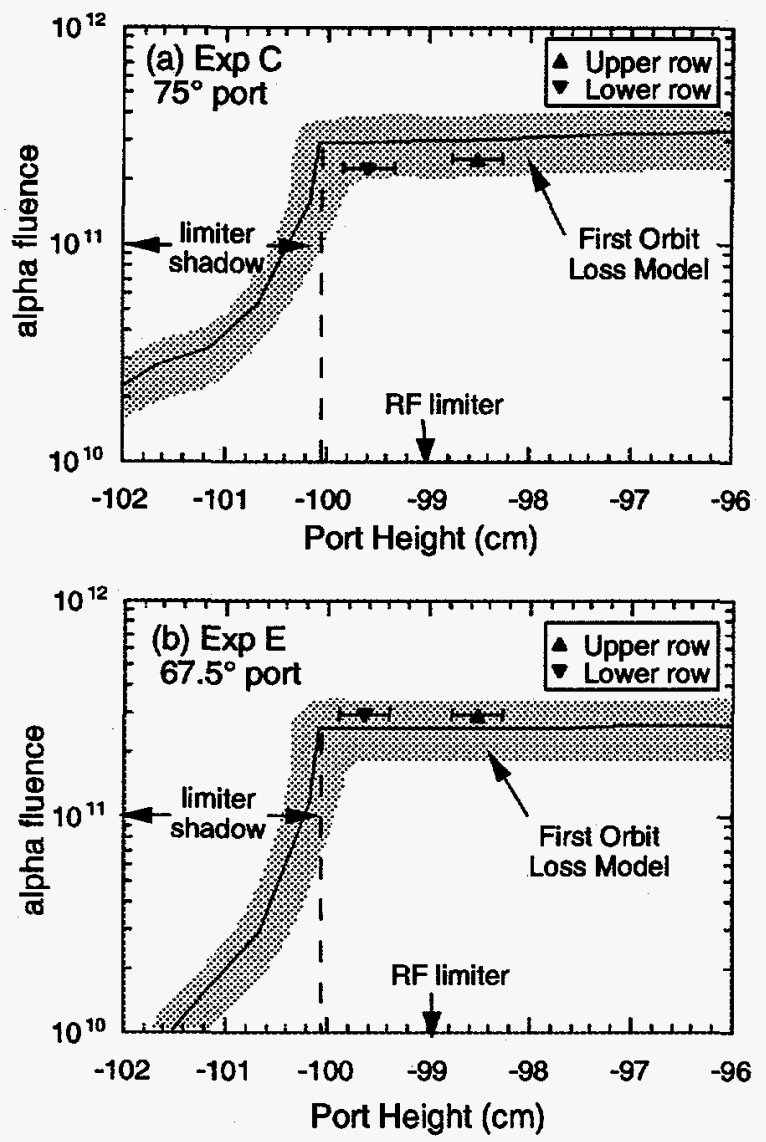

FIG. 19. $I_{p}=1.0 \mathrm{MA}-$ Alpha fluence for layers 4 through 9 as a function of port height for the upper and lower rows of the (a) $75^{\circ}$ port of exposure $C$, and (b) $67.5^{\circ}$ port of exposure $E$. The $R F$ limiter shadow begins $\sim 4 \mathrm{~mm}$ below the midplane of the lower row.

alpha collector (ie. $\sim$ a factor of 3 decrease for $\sim 1 \mathrm{~cm}$ radially outward movement near the RF limiter edge). There have not, however, been any indications of a partially thermalized loss to the $20^{\circ}$ scintillator detector.

\subsection{Uncertainties}

The minimum experimental uncertainty in the alpha fluence measurement is estimated to be equal to the minimum detectable fluence of $\sim \pm 5 \times 10^{8}$ alphas per sample [10]. For Figs. 9, 10, 19, and 20, where the fluence of layers 4 through 9 (6 layers) are summed, the minimum uncertainty is $\sim 1.2 \times 10^{9}\left(\approx \sqrt{6} \times 5 \times 10^{8}\right)$. For summed fluences $\geq 1.2 \times 10^{10}$ alphas, the experimental uncertainty is estimated to be $< \pm 10 \%$ [10], corresponding approximately to the height of a data point (ie. triangle symbol) on this semilog scale.

The $5^{\circ}$ uncertainty in the port orientation assigned to the data points in Figs. 9, 10, 15, and 16(a), corresponds to $\sim 2 \mathrm{~mm}$ on the circumference of the probe. This is
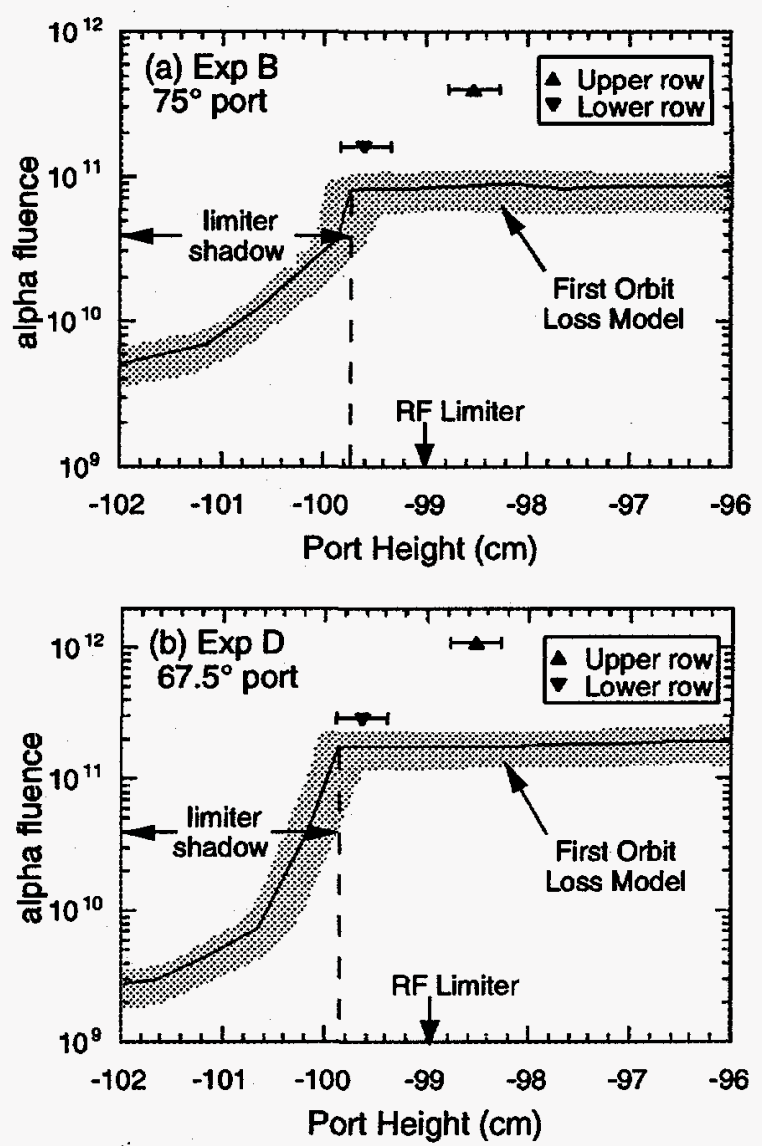

FIG. 20. $I_{p}=1.8 \mathrm{MA}$ - Alpha fluence for layers 4 through 9 as a function of port height for the upper and lower rows of the (a) $75^{\circ}$ port of exposure $B$, and (b) $67.5^{\circ}$ port of exposure $D$. The RF limiter shadow begins only $\sim 2 \mathrm{~mm}$ below the midplane of the lower row, possibly placing the lower row partially in the shadow. The decrease in the anomalous loss from the upper to lower row may be attributable to shadowing and/or a radial diffusive loss.

the maximum misalignment that might be expected from the method used to align the probe head onto its base, combined with the uncertainty in the base alignment with respect to the vessel.

The error in the first orbit loss model represented by the shaded region in Figs. 9, 10, 19, and 20, is based on several uncertainties. First of all, the source and current profiles used by the Lorentz ORBIT code are taken from TRANSP for one time during the flat top portion in the discharge. These profiles are used to represent the plasma for the entire duration of the shot. Performing the calculation for other times throughout the discharge results in less then $15 \%$ variation in the total fluence calculation. So the choice of a single time near the time of maximum fusion rate should introduce no more than $15 \%$ error. Another error in the first orbit loss model comes from converting the alpha collection fraction in alphas per neutron 
to total loss fluence by multiplying by the global neutron production. The global neutron measurements have an $\sim 5 \%$ error associated with them which is transferred to the alpha fluence calculation. Combining these uncertainties, along with other sources of uncertainty such as the accuracy of the TRANSP profiles, the overall minimum error in the first orbit loss model is estimated to be $\pm 30 \%$. This uncertainty is based on the modeling of the $75^{\circ}$ port. The $22.5^{\circ}$ port can have substantially more error since the main contribution of alphas to this port is from co-going alphas born near the edge of the plasma (eg. the $40^{\circ}$ orbit of Fig. 1), and is thus extremely sensitive to uncertainties in the source profile which can greatly affect the source term at the edge. The $75^{\circ}$ port fluences are dominated by alphas lost near the fattest banana orbit which pass closest to the magnetic axis where the source profile is peaked and are thus not as sensitive to the shape of the profile. The uncertainty due to the source profile was estimated using the results of a sensitivity analysis in which a parabolic to a power source profile was assumed: $S(r)=S_{0}\left(1-(r / a)^{2}\right)^{i_{s}}$, where $S(r)$ is the source profile as a function of minor radius $r, S_{0}$ is the alpha source term at the magnetic axis, $a$ is the edge minor radius, and $i_{s}$ is the source term peaking exponent. The Lorentz ORBIT calculated alpha collection fraction was evaluated for $i_{s}=8 \pm 1$, a common value for the peaking exponent with a reasonable uncertainty. Where the alpha collection fraction varied by more than $\pm 30 \%$, the higher uncertainty due to source profile sensitivity was used.

The first orbit model also incorporates a $\pm 5^{\circ}$ uncertainty in the port orientation which comes about due to uncertainties in the plasma current profile and modeling inaccuracies of the vacuum magnetic field in the Lorentz ORBIT code. As illustrated in Fig. 18, the first orbit model does not match the pitch angle distribution measured by the scintillator in the 1.0 MA case. Correcting this overestimate would roughly correspond to a shift of the model distributions to the left by $\sim 5^{\circ}$. The agreement between the data and the model for the 1.0 MA distributions, shown in Figs. 9 and 15, would be improved by such a shift. To account for this uncertainty, the first orbit model is given $a \pm 5^{\circ}$ spread.

The uncertainty in the left collection fraction data of Figs. 12 and 14 is based on comparing the maximum and minimum possible measured alpha fluences, based on the measurement uncertainty of the larger of $\pm 1.2 \times 10^{9}$ alphas or $\pm 10 \%$, for the left and right halves.

\section{DISCUSSION}

\subsection{Summary of Experimental Results}

Measurements of escaping alphas were made using the alpha collector probe for plasma currents of 1.0 and 1.8 MA. The He released from foil layers 4 through 9, which should be representative of first orbit lost alphas, was then compared to a first orbit loss model with respect to the total alpha fluence implanted into these foils, and the energy, pitch angle and radial distributions inferred from the measured loss. The comparison at 1.0 MA indicates that the measurement from layers 4 through 9 is consistent with the first orbit loss model for $3.5 \mathrm{MeV}$ alphas. The shallow layers (2 through 3 ), however, suggest that a small ( $\sim 1 / 3$ the fluence of first orbit loss), low energy $(<2.0 \mathrm{MeV})$ anomalous loss feature may be occurring at this plasma current.

The comparison at 1.8 MA reveals a partially thermalized loss with a total alpha fluence nearly an order of magnitude $(\approx 7 x)$ larger than that of the first orbit loss model and a wide energy distribution peaked at $\sim 2.5 \pm 0.3$ $\mathrm{MeV}$ (ie. $30 \%$ below the birth energy of $3.5 \mathrm{MeV}$ ). The pitch angle distribution of the inferred anomalous loss appears to occur in a narrow region above the passingtrapped boundary $\left(\chi_{f b} \approx 56^{\circ}\right)$ and is peaked at a toroidal pitch angle of $\sim 63^{\circ} \pm 7^{\circ}$. This anomalous loss drops by approximately a factor of 3 in magnitude from the upper row to the lower row of collimating ports (separated by $\sim 1.1 \mathrm{~cm}$ ), in contrast to the first orbit loss model which remains nearly constant between the two rows. There is no evidence of this anomalous loss on the $90^{\circ}$ lost alpha scintillator detector.

\subsection{Comparison of $1.8 \mathrm{MA}$ Anomalous Loss with Delayed Loss}

The anomalous results obtained with the alpha collector probe are qualitatively similar to an anomalous loss feature called 'delayed loss', which is not yet understood [17]. Delayed loss is observed with the lost alpha scintillator detectors for $\mathrm{DD}$ fusion products (ie. $3 \mathrm{MeV}$ proton, and $1 \mathrm{MeV}$ triton) in DD plasmas. Delayed loss, however, has not been observed with the scintillator detectors for DT alphas [1]. The following is a list of the observed characteristics of delayed loss and how they compare to the anomalous loss observed with the alpha collector probe.

1. Delayed loss is seen at the scintillator detector located $90^{\circ}$ poloidally below the outer midplane (ie. at the bottom of the TFTR vessel), but not at the $20^{\circ}$, $45^{\circ}$ nor $60^{\circ}$ detectors. This is consistent with the current results in that the measurements made with the 
alpha collector probe were only made at the bottom of the vessel.

2. The delayed loss to the $90^{\circ}$ scintillator detector has a strong dependence on the plasma major radius, being largest at the small major radius of $R=2.45 \mathrm{~m}$, and disappearing at $R>2.55 \mathrm{~m}$. Again, this is consistent with the current results in that the measurements made with the alpha collector probe were made in $R=2.45 \mathrm{~m}$ plasmas. The alpha collector was used only in these small plasmas to minimize probe heating by maximizing its distance from the plasma. The design does allow its use in up to $R=2.52 \mathrm{~m}$ plasmas, but no exposures were done at this radius.

3. The strength of delayed loss increases with respect to the first orbit loss with increased plasma current, becoming dominant above 1.8 MA. This is consistent with the result that only first orbit loss (with the exception of a small anomalous loss feature at low energy) was observed at 1.0 MA, but a large anomalous loss, in addition to first orbit loss, was observed at 1.8 MA using the alpha collector probe. The total loss (first orbit plus delayed loss) in DD at 1.8 MA as measured with the $90^{\circ}$ scintillator detector was about a factor of 2 above the expected first orbit loss [17], as compared to a factor of 7 for the alpha collector in DT at 1.8 MA.

4. The energy of delayed loss particles is about half (ie. $55 \pm 15 \%$ [17]) that of the prompt first orbit loss, as inferred from the gyroradius of its scintillatorimpact. This is consistent, within the energy resolution of the detectors, with the $\sim 70 \pm 10 \%$ of birth energy inferred for the anomalous loss of the alpha collector.

5. Delayed loss at $I_{p}=1.8 \mathrm{MA}$ occurs at a pitch angle approximately $10^{\circ}$ above that of the fattest banana orbit. This is roughly consistent with the anomalous loss pitch angle inferred to be $\sim 7^{\circ} \pm 7^{\circ}$ above the fattest banana pitch angle of $56^{\circ}$.

6. Delayed loss is delayed by $\sim 200 \pm 100 \mathrm{~ms}$ with respect to the usual first orbit loss, as can be seen most clearly at the beginning and end of neutral beam injection. The time resolution of the alpha collector is limited to a single discharge since it integrates alphas over an entire shot. Thus this feature of delayed loss can not be checked with the alpha collector. However, the inferred energy of $\sim 70 \pm 10 \%$ of the birth energy for the anomalous loss requires a delay of $\sim 100 \mathrm{~ms}$ for them to slow down to this energy (assuming an energy e-folding time of $\sim 200 \mathrm{~ms}$ ) consistent with delayed loss.

7. Delayed loss increases slowly with NBI power at a fixed plasma current. The alpha collector exposures were all done at an NBI power of $\sim 10 \mathrm{MW}$. Thus a comparison of the NBI power dependence of the two anomalous losses can not be made. The design of the collector probe does allow its use in full power discharges, making a beam power scan possible, but such a scan was not done.

8. For $R=2.45 \mathrm{~m}$ and $I_{p}=1.8 \mathrm{MA}$ plasmas, delayed loss to the $90^{\circ}$ scintillator detector is of the same order of magnitude as first orbit loss in DD plasmas, but is absent in DT plasmas. The anomalous loss to the alpha collector appears to be about a factor of 6 times larger than first orbit loss in DT plasmas. The alpha collector is not capable of detecting DD fusion products. The lack of detectable levels of delayed loss on the scintillator detector in DT plasmas but apparently large levels in the alpha collector is most likely due to the different radial positions of the two probes. The lower row of the alpha collector extends nearly $1 \mathrm{~cm}$ farther into the vessel than does the pinhole aperture of the $90^{\circ}$ scintillator detector. The strong radial dependence of the anomalous loss, seen in the upper/lower row comparisons of Fig. 20, may be sufficient to make it an insignificant contribution to the loss observed by the scintillator detector. Their different toroidal positions relative to toroidal asymmetries in the vessel such as limiters and the path of neutral heating beams may also be a factor.

It may be significant to note that the delayed loss features changed significantly when the $90^{\circ}$ scintillator detector was repositioned after the 1990 run to accommodate the installation of a new poloidal RF limiter [17]. Previously, the detector aperture was located about $4 \mathrm{~cm}$ radially outside (ie. below) and about $120^{\circ}$ toroidally from the edge of the nearest limiter. The new limiter was installed only $45^{\circ}$ toroidally from this detector which forced a relocation of its aperture to only about $1 \mathrm{~cm}$ below the edge of this new limiter to avoid shadowing of the aperture [18]. After the repositioning, the delayed loss feature increased in magnitude (by a factor of $\sim 4$ at $I_{p}=1.8 \mathrm{MA}$ ) and peaked at a pitch angle closer to the passing-trapped boundary. The implication was that after its repositioning, the $90^{\circ}$ detector collected more anomalous delayed loss at low pitch angles than it had previously, presumably because these ions had not reached the aperture of the detector in the 1990 run. The first orbit loss features remained essentially unchanged between the two runs. 


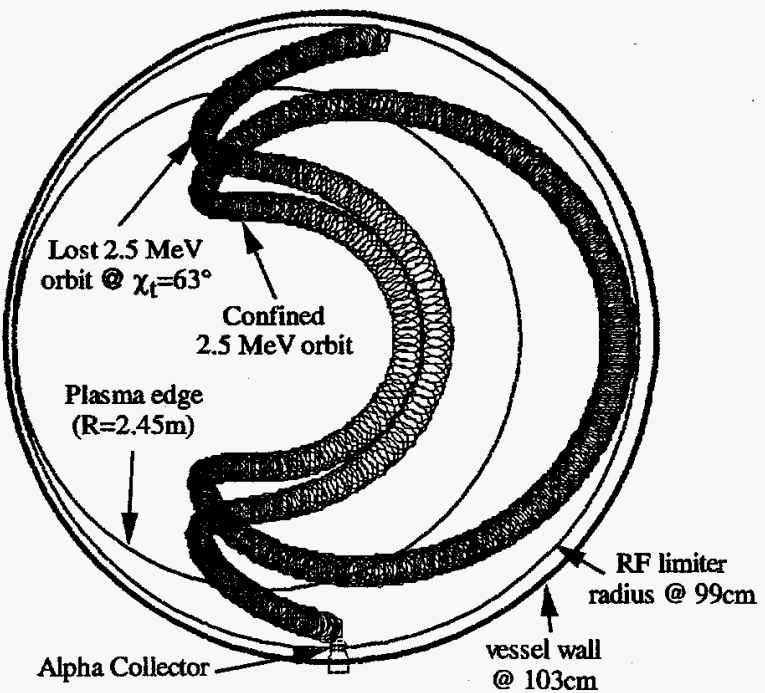

FIG. 21. Trajectories in a $R=2.45 \mathrm{~m}, I_{p}=1.8 \mathrm{MA}$ plasma of an anomalous loss orbit $\left(2.5 \mathrm{MeV}, \chi_{t}=63^{\circ}\right)$ that strikes the detector at the bottom of the TFTR vessel and a marginally confined orbit with the same parameters that just misses the $R F$ limiter at the outer midplane. The banana tips of the two orbits are displaced by $\sim 17 \mathrm{~cm}$.

This demonstrates the fact that delayed loss is extremely sensitive to the relative positioning of a detector at $90^{\circ}$ and the obstacles that fusion products may encounter such as RF limiters.

The similarities between delayed loss and the anomalous loss observed with the alpha collector imply that they may be due to the same loss mechanism. In section 5.4 some of the possible loss mechanisms are considered.

\subsection{Trajectories of anomalous loss orbits}

The trajectory of an alpha particle's last orbit prior to intersection with the detector can be calculated using the Lorentz ORBIT code. Fig. 21 shows the last orbit traced backwards in time from the alpha collector at the bottom of the vessel to an RF limiter at the top of the vessel for an alpha particle at $E=2.5 \mathrm{MeV}$ and $\chi_{t}=63^{\circ}$ (the parameters inferred for the anomalous loss) for the $\mathrm{R}=2.45 \mathrm{~m}, I_{p}=1.8 \mathrm{MA}$ discharge of exposure B. Also shown in Fig. 21 is a marginally confined orbit of the same energy which was started with an upward vertical displacement of the lower banana tip of $\sim 17 \mathrm{~cm}$ with respect to the last orbit. The outer leg of this confined orbit just barely misses the projection of the RF limiter (centered at $R=2.61 \mathrm{~m}$ with a minor radius of $0.99 \mathrm{~m}$ ) near the outer midplane. Thus, confined orbits with banana tips just below this one intersect the wall just below the outer midplane. Therefore, the anomalous loss orbits detected at $90^{\circ}$ could be brought there by a relatively large vertical step on the last bounce of a previously confined trapped orbit, while smaller vertical steps would cause the loss to occur nearer the outer midplane, as in stochastic ripple diffusion [19].

A detailed study of many such anomalous loss orbits has shown that the vertical displacement of the lower banana tip required for a previously confined orbit to reach the alpha collector at the bottom of the vessel (for $E=2.2$ to $2.8 \mathrm{MeV}$ alpha orbits within $\chi_{t}=56^{\circ}-$ $70^{\circ}$ ) is at least $15 \mathrm{~cm}$. Such a large step-size, however, is inconsistent with the observed radial dependence of the anomalous loss. The $1.1 \mathrm{~cm}$ separation between the upper and lower rows of ports is much less than the required step-size of $>15 \mathrm{~cm}$. Thus there should be little variation in the alpha fluence between the rows, but measurements show a factor of 3 difference between the upper and lower row.

Small step-size radial diffusion could explain the observed radial dependence except for the fact that the corresponding high energy alpha orbits cannot make it to the bottom of the vessel, having been scraped off at the midplane. As can be inferred from Fig. 21, the probe would have to be placed $\sim 20 \mathrm{~cm}$ further into the vessel to intercept a marginally confined alpha orbit with the anomalous loss parameters. A smaller banana width could allow an alpha orbit to strike the bottom of the vessel first, but this would require an alpha energy of $\sim 0.3 \mathrm{MeV}$, less than the minimum detectable energy of the alpha collector. But, if for the sake of argument, one assumes that there are orbits that strike the bottom of the vessel first as they diffuse radially outwards, then the radial dependence of the anomalous loss can be used to calculate a diffusive step-size. Assuming that at every bounce the particles walk randomly with a step-size of $b$, and that they will be scraped-off by an obstacle (such as the RF limters or the probe head) with a probability $P_{l}$, the alpha flux at a distance $x$ behind the obstacle is $I(x)=I_{0}\left(1-P_{l}\right)^{N}$, where $I_{0}$ is the alpha flux at $x=0$, and $N=(x / b)^{2}$ is the number of bounces needed for the particle to randomly walk the distance $x$ [20]. The probability of scrape-off is estimated to be the ratio of the toroidal extent of the obstacles above $x$ to the toroidal circumference of the vessel. It can easily be shown that the scrape-off on the probe head is insignificant compared to the scrape-off on the RF limiters. For the lower row of ports, with $x=0.6 \mathrm{~cm}$ and $P_{l} \approx 0.13$ (eight limiters that each have a toroidal extent above $x$ of $\sim 27 \mathrm{~cm}$ ), the factor of 3 between rows results in a step-size of $\sim 0.2 \mathrm{~cm}$. Diffusion at this step-size down to the location of the scintillator detector, with $x=1.2 \mathrm{~cm}$ and $P_{l} \approx 0.19$ (toroidal extent of each limiter above $x$ increased to $\sim 39 \mathrm{~cm}$ ) occurs with a flux reduction by a factor of $\sim 2000$ with respect to the upper row of ports, 
consistent with the absence of anomalous loss to the $90^{\circ}$ scintillator detector.

A step-size of $0.2 \mathrm{~cm}$ per bounce corresponds to a diffusion coefficient, $D=b^{2} / \tau_{b}$, of $\sim 0.4 \mathrm{~m}^{2} / \mathrm{s}$, where the bounce period $\tau_{b} \approx 10 \mu \mathrm{s}$. This results in a time scale for diffusion to the wall $\tau_{D} \approx a^{2} / 4 D$ of $\sim 0.6 \mathrm{~s}$. Assuming all the alphas diffuse at this rate, $\sim 30 \%$ of the alphas should be lost to the wall within one energy e-folding time. However, small step-size diffusion to the bottom of the TFTR vessel probably isn't realistic since it is not consistent with high energy orbits that tend to scrape-off near the midplane. A larger step-size would result in a larger global loss, however, there would likely be a threshold condition such that not all of the alphas are included in the diffusive process. Thus, without knowing the loss mechanism, it is difficult to estimate the global loss associated with the observed anomalous loss.

The large step-size of $17 \mathrm{~cm}$ inferred from the orbits of Fig. 21 is consistent with the $15 \mathrm{~cm}$ that was estimated in the same manner in Ref. [17] for delayed loss of $1.5 \mathrm{MeV}$ (half the birth energy) DD fusion protons to the $90^{\circ}$ scintillator detector for a $\mathrm{R}=2.45 \mathrm{~m}, I_{p}=2.0 \mathrm{MA}$ discharge. Thus the arguments as to the unlikeliness of pitch angle scattering, TF ripple, and MHD as possible causes of delayed loss [17] also apply to the 1.8 MA anomalous loss observed with the alpha collector probe, and will thus only be briefly summarized in the next section. Several new possibilities that attempt to explain the anomalous loss are also considered in the next section.

\subsection{Possible anomalous loss mechanisms}

A model attempting to explain the mechanism responsible for the anomalous loss observed with the alpha collector would have to be consistent with the following features:

a) Absence of anomalous loss on the $90^{\circ}$ lost alpha scintillator detector in DT.

b) $I_{p}$ dependence - occurs at 1.8 MA but not at 1.0 MA.

c) Total fluence - $\approx 6$ times larger than first orbit loss.

d) Energy distribution - peak $\sim 2.5 \pm 0.3 \mathrm{MeV}$ inferred from shallow range distribution.

e) Pitch angle distribution - peak $\sim 7 \pm 7^{\circ}$ above the passing-trapped boundary, and most likely concentrated in narrow region above this boundary.

f) Radial dependence - factor of 3 decrease from upper to lower row (separated by $1.1 \mathrm{~cm}$ ).
The anomalous loss mechanisms described in the following sections are summarized in Table II with respect to their consistency with the observed loss features listed above.

\subsubsection{Collisional loss}

The $\sim 0.2 \mathrm{sec}$ time delay observed on the $90^{\circ}$ scintillator detector for delayed loss suggests a classical collisional loss mechanism, since this time is on the order of the slowing down time for fusion products [21]. Although large pitch angle scattering is capable of causing a confined orbit to become lost, it is too infrequent to be of significance, occurring on a time scale of $\sim 10 \mathrm{sec}$ [22].

Small pitch angle scattering of barely passing alphas into the first orbit loss cone can generate a diffusion of alphas across the passing-trapped boundary [22]. The alphas that are subsequently lost should be marginally trapped, ie. they should appear at the detector at the pitch angle of the passing-trapped boundary $\left(\sim 56^{\circ}\right.$ at $\left.1.8 \mathrm{MA}\right)$. However, delayed loss is seen to occur at pitch angles clearly above the passing-trapped boundary. This also appears to be the case with the alpha collector anomalous loss at 1.8 MA (see section 4.3.2.). Furthermore, models consistently predict small loss fractions for collisional loss relative to first orbit loss. For instance, Ref. [21] reported a TRASNP prediction for a $\mathrm{R}=2.45 \mathrm{~m}, I_{p}=1.6 \mathrm{MA}$ TFTR discharge of a global loss due to collisions of alphas of only $0.35 \%$, which was only $5 \%$ of the calculated first orbit loss fraction for that shot.

\subsubsection{Toroidal field (TF) ripple effects}

There are at least two different mechanisms through which TF ripple can cause radial transport of fast ions. Stochastic ripple diffusion (SRD) [23] is a collisionless process which produces a radial step near the banana tip of those trapped particles that meet a particular threshold criteria. The maximum vertical step-size for $2.5 \mathrm{MeV}$ alphas in the conditions of discharge $B$ can be shown to be $\approx 5 \mathrm{~cm}$, in a manner similar to the calculation of Ref. [17] that estimated a $\approx 3.5 \mathrm{~cm}$ maximum step-size for $1.5 \mathrm{MeV}$ protons in a 2 MA plasma. Therefore, even if a trapped particle passes through two banana tips before passing near the midplane, it can only gain up to a maximum of $10 \mathrm{~cm}$ outward displacement, significantly less than the $17 \mathrm{~cm}$ necessary to reach the alpha collector at the bottom of the vessel (see section 5.3). This relatively small step-size is such that almost all of the SRD loss should be localized within $30^{\circ}$ of the outer midplane [19].

A synergistic enhancement of fast ion diffusion has been found for SRD with collisions [11]. This effect is 


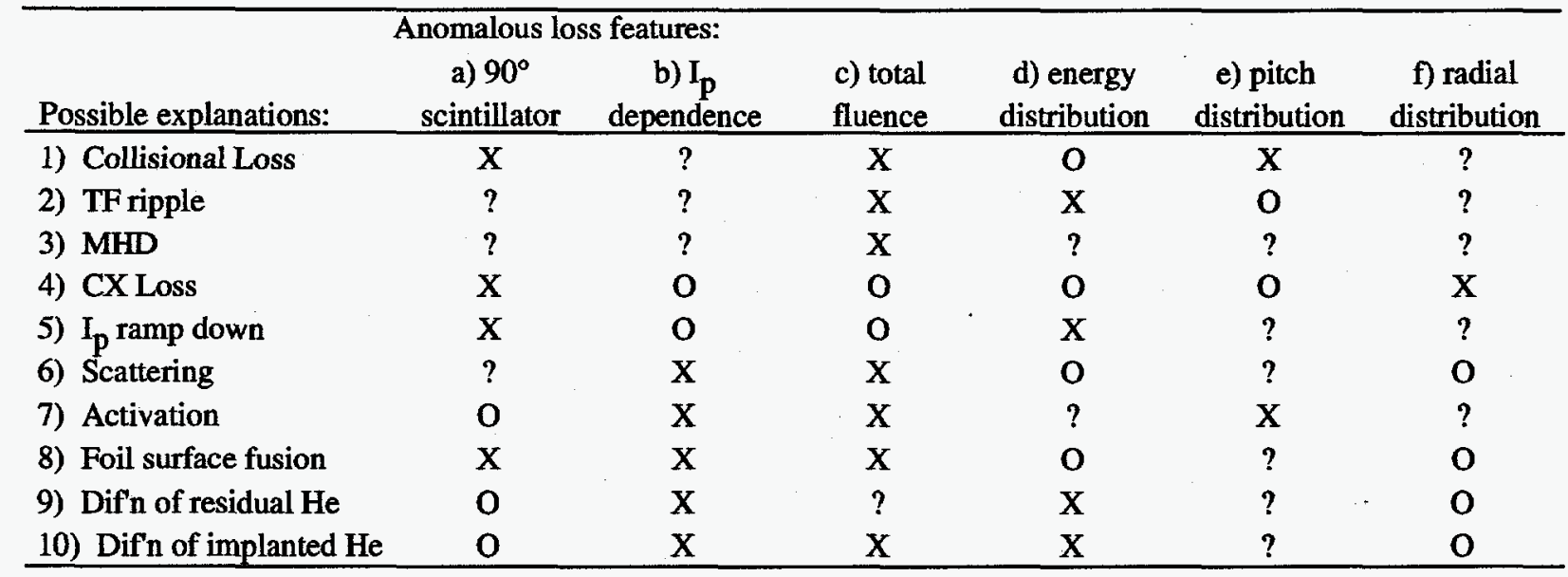

$\mathrm{X}$ - Inconsistent, $\mathrm{O}$ - Consistent, ? - Undecided

simulated using the Hamiltonian guiding center drift orbit Monte Carlo code, gc-ORBIT [24] (not to be confused with the Lorentz ORBIT code). Under the conditions of the 1.0 MA discharges, this code predicts a global alpha loss of $\sim 25 \%$. Only $\sim 15 \%$ of this global loss is 'delayed' (ie. occurring below $3.5 \mathrm{MeV}$ ). For the conditions of the 1.8 MA discharges, this code predicts a global alpha loss of $\sim 4 \%$, of which only $\sim 21 \%$ is 'delayed'. Thus, the synergistic enhancement is not of sufficient magnitude to make the 'delayed' losses at 1.8 MA much more significant than they are at 1.0 MA. Furthermore, these 'delayed' losses are clearly peaked poloidally within $30^{\circ}$ below the outer midplane.

The other TF ripple effect that can cause radial transport of fast ions is superbanana trapping inside the ripple wells [17]. Particles lost through ripple well trapping would have pitch angles very close to $90^{\circ}$, causing most of them to be self-shadowed by the probe head, as shown in Fig. 17. Note that a true pitch angle of $90^{\circ}$ corresponds to a toroidal pitch angle of $\sim 85^{\circ}$ due to the $\sim 5^{\circ}$ offset between true and toroidal pitch angles (see section 4.3.2.). The detection of the partially thermalized anomalous loss in the foil stack in the $30^{\circ}$ collimating port of exposure B (Fig. 10(b)) indicates that the anomalous loss extends down to toroidal pitch angles below $75^{\circ}$ (see section 4.3.2.). Furthermore, the left collection fraction analysis of section 4.3.2. gives an estimate of $63^{\circ} \pm 7^{\circ}$ for the toroidal pitch angle of the anomalous loss, slightly smaller than the $\sim 70^{\circ}$ measured by the $90^{\circ}$ scintillator detector for delayed loss. Ref. [17] concluded that it was unclear how particles lost through this mechanism could arrive at the detector with pitch angles this small. Most importantly, a loss of ripple well trapped particles at a true pitch angle of $\sim 90^{\circ}$ would have a better likelihood of implanting into the foils in the $120^{\circ}$ port than in the $75^{\circ}$ port because the $75^{\circ}$ port has a larger self-shadowing effect. However, the anomalous loss is not detected in the $120^{\circ}$ foils. Thus, it seems unlikely that ripple well trapping could provide an explanation of the anomalous loss.

\subsubsection{MHD effects}

The two mechanisms by which magnetic perturbations due to MHD activity can cause radial transport of high energy particles are considered with respect to delayed loss in Ref. [17]. These mechanisms are the parallel drift of the ion along radially perturbed field lines, and the perpendicular drifts across the field lines. Both the former mechanism and a non-resonant interaction of the latter, required an unrealistically large magnetic perturbation of $\tilde{B}_{r} / B_{T} \approx 10^{-2}$ to achieve a $10 \mathrm{~cm}$ step-size [17], where $\tilde{B}_{r}$ is the local radial magnetic perturbation. The magnitude of normal magnetic perturbations inside plasmas is generally $\tilde{B}_{r} / B_{T} \approx 10^{-4}[25,26]$. Similarly, orbits resonant with the perturbation required the largest MHD perturbations in TFTR [17]. Thus it was concluded that the effects of some hidden MHD activity during seemingly MHD quiescent plasmas cannot easily explain the large last step necessary to bring the escaping orbit to the $90^{\circ}$ detector [17]. This conclusion applies equally well to the MHD quiescent discharges conducted for the alpha collector exposures as it did to discharges examined for delayed loss.

\subsubsection{Loss of $\mathrm{He}^{+}$from $\mathrm{CX}$ with impurities or NBI neu- trals}

Another possibility is that fully stripped alphas charge exchange with partially stripped impurities producing 


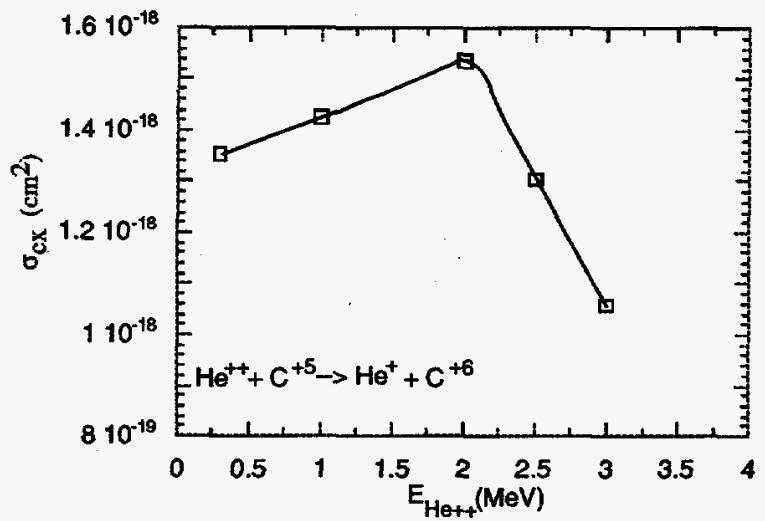

FIG. 22. Calculated single electron capture cross section for the $\mathrm{He}^{++}+\mathrm{C}^{+5}$ collision system.

singly ionized alphas through:

$$
H e^{++}+A^{+q} \rightarrow H e^{+}+A^{+q+1}
$$

where $A^{+q}$ are plasma impurity ions. The singly ionized alpha particle then has twice the gyroradius it had before charge exchange, resulting in a doubling of its banana width. The orbits of previously confined alphas may suddenly transition to prompt loss trajectories that take some of the alpha particles to the detector.

Ref. [27] concluded that for a $0.1 \%$ low charge state impurity concentration of oxygen (ie. $\sim 1 \times 10^{11} \mathrm{~cm}^{-3}$ ) in TFTR that the average time for single electron capture for $2.0 \mathrm{MeV} \mathrm{He}++$ is $\approx 5 \mathrm{~ms}$. This is much less than the $\sim 200 \mathrm{~ms}$ alpha slowing down time and hence should cause a net diffusion to the container walls.

With the addition of carbon tiles, the main impurity in TFTR is now carbon, not oxygen. At Zeff $\approx 1.5$ the impurity concentration for carbon is $\approx 5 \%$, with roughly the same radial profile as the electron density. At electron temperatures $\sim 10 \mathrm{KeV}$ most of this carbon is fully stripped $\left(C^{+6}\right)$ in the core. PPPL's MIST code solves for the density of ions in each charge state using atomic physics appropriate for these low-density hightemperature plasmas [28]. MIST predicts a $\mathrm{H}$-like carbon $\left(C^{+5}\right)$ concentration of $\sim 5 \times 10^{9} \mathrm{~cm}^{-3}\left(\sim 0.01 \% n_{e}\right)$ in the core, which increases by a factor of $\sim 30$ at the cooler plasma edge. The lower charge states of carbon are present in the core at concentrations reduced by a factor 1000 or more in relation to $C^{+5}$. The charge exchange cross section calculated for the $\mathrm{He}^{++}+\mathrm{C}^{+5}$ collision system using the classical-trajectory Monte-Carlo method [29] is seen in Fig. 22 to be $\approx 3 \times 10^{-18} \mathrm{~cm}^{2}$ for alphas at $2.5 \mathrm{MeV}$. This classical treatment may not be appropriate in the energy range being considered due to unphysical capture to deeply bound states that only exist classically resulting in cross sections that may be unrealistically large by up to a factor of about 5 at $\sim 2.5 \mathrm{MeV}$. Work is progressing in this area to substantiate these values $[30,31]$. For the purposes of this discussion, the values obtained in Fig. 22 will be used. This results in a collision period for charge exchange of $2.5 \mathrm{MeV}$ alphas in the core of $\tau_{c x} \approx 150 \mathrm{~ms}$, which is of the same order as the alpha energy e-folding time, and a period of $\tau_{c x} \approx 5 \mathrm{~ms}$ at the edge. Charge exchange can therefore be a significant factor in the evolution of alpha orbits as they slow down, assuming that these cross sections are reasonable.

Fig. 23 illustrates possible orbit transitions due to charge exchange in a 1.8 MA plasma (exposure B) that can cause previously confined alphas to strike the detector at the bottom of the vessel. Fig. 23(a) depicts a 2.5 $\mathrm{MeV}$ trapped alpha that picks up an electron through charge exchange as it crosses the outer midplane on its counter-going leg and is subsequently lost to the detector. Fig. 23(b) depicts a $2.5 \mathrm{MeV}$ counter-going passing alpha that also charge exchanges as it crosses the outer midplane and is subsequently lost to the detector. A similar figure would show that co-going passing alphas that charge exchange as they cross the inner midplane can also be subsequently lost to the detector on a co-going trajectory.

Fig. 24 illustrates what happens when alphas charge exchange on the opposite side of orbits similar to those of Fig. 23. Fig. 24(a) shows that a $2.5 \mathrm{MeV}$ trapped alpha charge exchanging as it crosses the outer midplane on its co-going leg results in an orbit that passes closer to the magnetic axis. Similarly, Fig. 24(b) shows a $2.5 \mathrm{MeV}$ counter-going passing alpha that charge exchanges as it crosses the inner midplane also resulting in slight inward radial transport. Again, a similar figure would show that co-going passing alphas that charge exchange as they cross the outer midplane also transport inwards. These particles are quickly reionized by the background plasma within a few poloidal transits since the collision period for reionization in the core of $\sim 5 \mu \mathrm{s}$ [31] is on the order of the bounce frequency of $\sim 10 \mu \mathrm{s}$. The lost orbits of Fig. 23, however, reach the detector within $\sim 3 \mu \mathrm{s}$, spending very little time in the core where the plasma density is peaked, and thus have a much lower probability of being reionized.

Figs. 23 and 24 represent the extremes in the orbit transitions possible through charge exchange. Charge exchange at other locations along an orbit results in less radial transport. A series of successive charge exchanges and reionizations could result in a radial diffusive process. However, as discussed in section 5.3, the large radial stepsize needed to bring the orbits to the detector is inconsistent with the factor of 3 reduction in fluence between the upper and lower rows of ports. It is not obvious how 

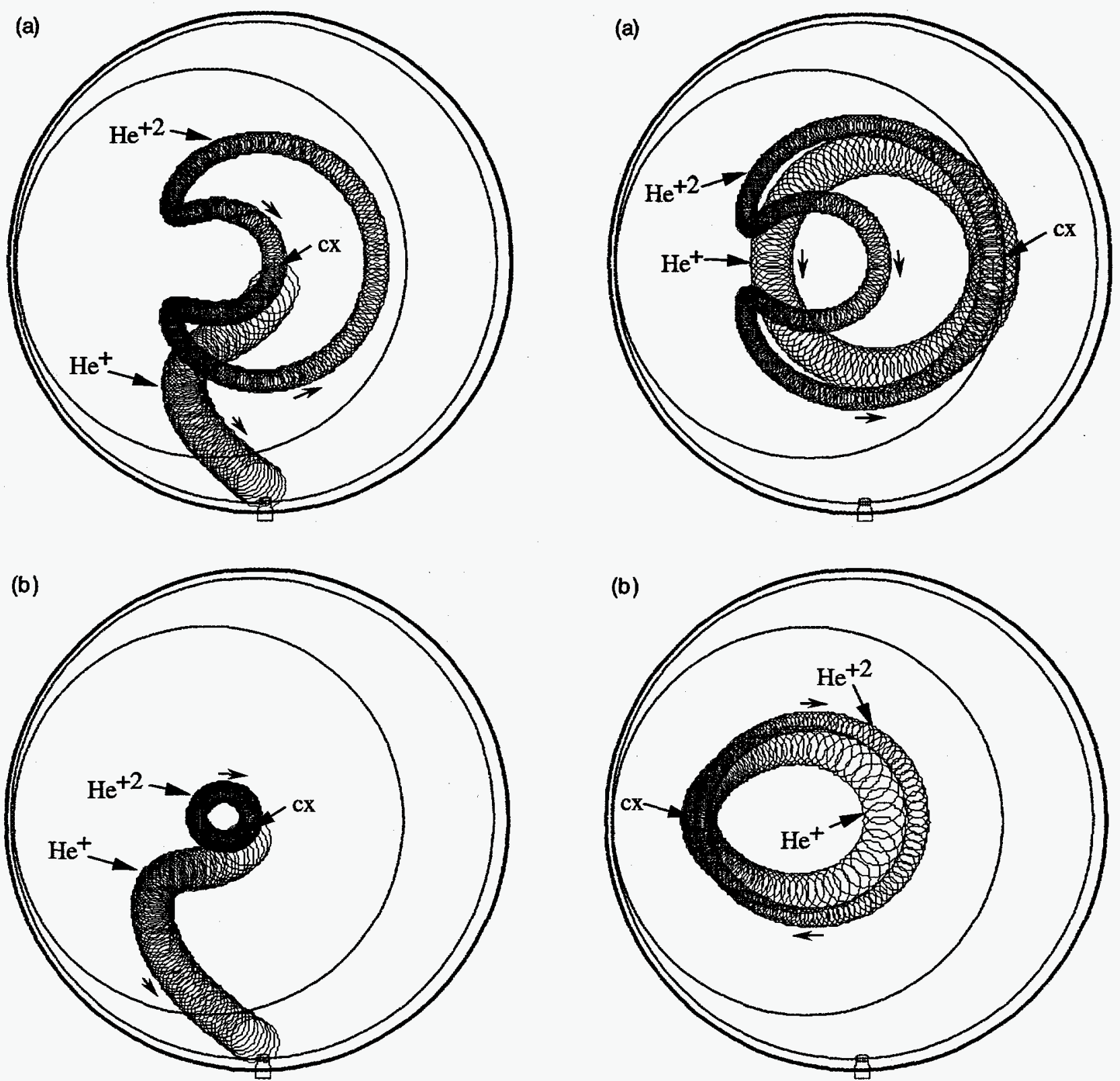

FIG. 23. $2.5 \mathrm{MeV}$ alpha orbit transitions that take alphas to the detector at $90^{\circ}$ in $R=2.45 \mathrm{~m}, I_{p}=1.8 \mathrm{MA}$ plasmas for alphas charge exchanging $(c x)$ at (a) the outer-midplane crossing point of the counter-going leg of a trapped particle $(R=270 \mathrm{~cm}$, $\left.\chi_{t}=63.5^{\circ}\right)$, and $(b)$ the outer-midplane crossing point of a counter-going passing particle $\left(R=260 \mathrm{~cm}, \chi_{t}=65.3^{\circ}\right)$. Small arrows show direction of guiding center motion.

charge exchange loss could account for the radial dependence and absence of anomalous loss on the lost alpha scintillator detectors in DT. The larger downward drifts associated with the larger gyroradius of the $\mathrm{He}^{+}$should make shadowing by the nearby RF limiters less effective, and hence allow the charge exchange lost particles to easily reach the lower row of collimating ports on the alpha

FIG. 24. $2.5 \mathrm{MeV}$ alpha orbit transitions in $R=2.45 \mathrm{~m}$, $I_{p}=1.8 \mathrm{MA}$ plasmas for alphas charge exchanging (cx) at (a) the outer-midplane crossing point of the co-going leg of a trapped particle $\left(R=320 \mathrm{~cm}, \chi_{t}=124^{\circ}\right)$, and $(b)$ the innermidplane crossing point of a counter-going passing particle $\left(R=200 \mathrm{~cm}, \chi_{t}=70^{\circ}\right)$, both resulting in inwards radial transport.

collector and the scintillator detector.

Fig. 25 is a plot of toroidal pitch angle vs. $r / a$ for the outer midplane crossing point of counter-going particles in a 1.8 MA plasma. Both of these quantities are nearly conserved during an electron capture. The solid lines designate the boundaries for fully stripped $3.5 \mathrm{MeV}$ alphas. The Passing-Trapped boundary separates counter-passing 


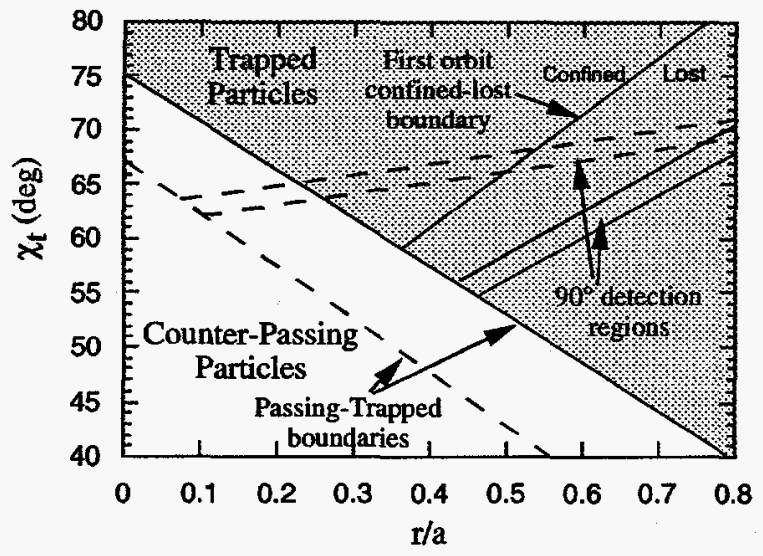

FIG. 25. Distribution of the different orbit classes for $R=2.45 \mathrm{~m}, I_{p}=1.8 \mathrm{MA}$ plasma in toroidal pitch angle versus their outer midplane crossing point for counter-going particles. Solid boundaries are for fully stripped $3.5 \mathrm{MeV}$ alphas. Dashed boundaries are for singly ionized $2.5 \mathrm{MeV}$ alphas representative of the anomalous loss.

orbits, which occur at low pitch angle or low minor radius, from trapped orbits (shaded region). The First Orbit Confined-Lost boundary separates trapped confined orbits, which occur in the trapped region at high pitch angle or low minor radius, from trapped lost orbits. Within the region of trapped lost orbits is a subset of orbits, labeled as the $90^{\circ}$ detection region, that intersect the detector at $90^{\circ}$ below the midplane. The dashed lines designate the boundaries for singly ionized $2.5 \mathrm{MeV}$ alphas. All of the trapped $2.5 \mathrm{MeV}$ singly ionized alphas in this plot are lost in one poloidal orbit, thus there is no First Orbit Confined-Lost boundary for these particles. The region of $2.5 \mathrm{MeV}$ singly ionized alphas that intersect the vessel at $90^{\circ}$ below the midplane are seen to correspond to previously first orbit confined (ie. counter-passing and trapped confined orbits) $3.5 \mathrm{MeV}$ fully stripped alphas all the way out to $r / a \approx 0.5$, within which over $90 \%$ of the alphas are born for a standard alpha source distribution (ie. parabolic to the eighth power). Therefore, nearly all of the $2.5 \mathrm{MeV}$ singly ionized alpha orbits that strike the $90^{\circ}$ detector were previously confined.

An examination of alpha orbits for a 1.0 MA plasma (producing a figure analogous to Fig. 25) has shown that all of the $2.5 \mathrm{MeV}$ singly ionized alphas that intersect the vessel at $90^{\circ}$ below the midplane correspond to previously first orbit lost $3.5 \mathrm{MeV}$ fully stripped alphas. Therefore, there is no reservoir of confined alphas at $1.0 \mathrm{MA}$ available to take part in this loss mechanism, explaining the absence of the anomalous loss at 1.0 MA.

The total fluence from this loss mechanism is estimated by modeling it as a new source of alphas at a birth energy of $2.5 \mathrm{MeV}$, carrying a single charge, with an isotropic velocity distribution and the same radial source profile as the $3.5 \mathrm{MeV}$ birth energy alphas, allowing the use of the Lorentz ORBIT code. The predicted fluence of $2.5 \mathrm{MeV}$ charge exchanged alphas (ie. $\mathrm{He}^{+}$) to the $75^{\circ}$ port of exposure $B$ is a factor of 2 larger than the first orbit loss prediction under these assumptions. This falls short of the ratio of measured anomalous loss to predicted first orbit loss of 6 by a factor of 3 . However, a flattening of the radial source profile from parabolic to the eighth to pure parabolic is sufficient to account for the full factor of 6. Flattening of the profile might be expected due to the peaking of the $C^{+5}$ density profile on the edge of the plasma, making charge exchange more likely to occur there. Also, the assumption of an isotropic velocity distribution may affect the calculation.

A similar calculation (also assuming a new source of isotropic $2.5 \mathrm{MeV} \mathrm{He}^{+}$with the same source profile as the $3.5 \mathrm{MeV} \mathrm{He}^{++}$) using the gc-ORBIT code (see section 5.4.2) to predict the global first orbit loss (rather than to a particular detector) in exposure B yields $22.2 \%$ global loss, a factor of $\sim 8$ larger than the $2.7 \%$ predicted for first orbit loss of $3.5 \mathrm{MeV}$ alphas. And the loss to a poloidally localized region of the wall about $90^{\circ}$ where the detector is located is $\sim 6$ times larger for $2.5 \mathrm{MeV}$ $\mathrm{He}^{+}$than for $3.5 \mathrm{MeV} \mathrm{He}^{++}$. The factor of 3 difference between the Lorentz ORBIT and gc-ORBIT predictions at $90^{\circ}$ is probably due to the different projections that the singly and doubly charged alpha orbits that are collected by the detector would make on the wall. This implies that the $2.5 \mathrm{MeV} \mathrm{He}^{+}$orbits collected by the detector would project onto an area of the wall $\sim 3$ times smaller than the $3.5 \mathrm{MeV} \mathrm{He}^{++}$, owing to the fact that the larger downward drift of the $2.5 \mathrm{MeV} \mathrm{He}^{+}$causes them to strike the wall at angles closer to normal incidence thus creating a smaller projection. While these are just rough estimates of charge exchange loss, they do indicate that this mechanism might be capable of generating losses on the order of magnitude of the observed anomalous loss.

To get a more realistic estimate of the total loss it will be necessary to develop or modify a guiding center following code to include charge exchange and reionization as a function of alpha energy during the slowing down process. This code would also need to take into account the radial dependence of the charge exchange and reionization probabilities since charge exchange is more likely to occur at the edge where the $C^{+5}$ density profile is peaked, and reionization is more likely to occur in the core where the plasma density profile is peaked.

The pitch angle distributions for first orbit loss of $3.5 \mathrm{MeV} \mathrm{He} e^{++}$and charge exchange loss of $2.5 \mathrm{MeV}$ $\mathrm{He}^{+}$calculated by the Lorentz ORBIT code as described above are shown in Fig. 26. The peak of the $2.5 \mathrm{MeV} \mathrm{He} e^{+}$ 


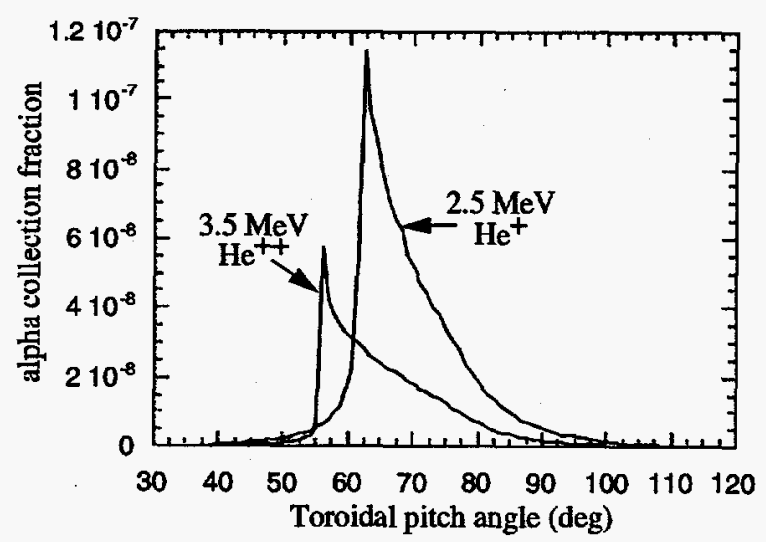

FIG. 26. Toroidal pitch angle distributions calculated at the detector for $3.5 \mathrm{MeV}$ fully stripped alphas and $2.5 \mathrm{MeV}$ singly ionized alphas in a $R=2.45 \mathrm{~m}, I_{p}=1.8 \mathrm{MA}$ plasma, assuming the same source profile and an isotropic velocity distribution in each case.

distribution occurs $\sim 6^{\circ}$ higher in toroidal pitch angle than it does for the first orbit loss of $3.5 \mathrm{MeV} \mathrm{He}^{++}$. This is consistent with the inference that the anomalous loss peaks at a pitch angle $\sim 7^{\circ} \pm 7^{\circ}$ above the passing-trapped boundary for first orbit loss (see section 4.3.2.). It is not clear that the shape of the pitch angle distribution for charge exchange loss would be the same as that calculated by treating it as a first orbit loss of $2.5 \mathrm{MeV} \mathrm{He} e^{+}$, but the peak is likely to remain fixed at $62^{\circ}$ since this corresponds to the passing-trapped boundary for $2.5 \mathrm{MeV} \mathrm{He}^{+}$in a 1.8 MA plasma.

The energy distribution of charge exchange lost particles can be estimated by calculating the probability of charge exchange throughout the slowing down process. The probability for charge exchange at energy $E$, over a small period, $\Delta t$, such that $E$ is fairly constant is:

$P_{c x}=1-e^{-\Delta t / \tau_{c x}(E)}$

where the collision period for charge exchange, $\tau_{c x}$, is calculated as a function of energy as the alphas slows down using Fig. 22 . The time to slow down by $0.25 \mathrm{MeV}$ increments is estimated from:

$$
\frac{d E}{d t}=-\frac{E}{\tau_{s d}} \approx \frac{\Delta E}{\Delta t}
$$

where $\tau_{s d}$ is the slowing down time (ie. energy e-folding time) in the core. Reionization is neglected such that once an alpha undergoes charge exchange it is assumed that it is no longer available to charge exchange at a lower energy. The resulting energy distribution is shown in Fig. 27 for three different slowing down times. The inferred energy of the peak of the anomalous loss of $2.5 \pm 0.3 \mathrm{MeV}$ is seen to be consistent with $\tau_{s d}=200 \pm 50 \mathrm{~ms}$. The higher anomalous loss energy inferred from exposure $\mathrm{D}(2.7 \pm 0.3 \mathrm{MeV})$

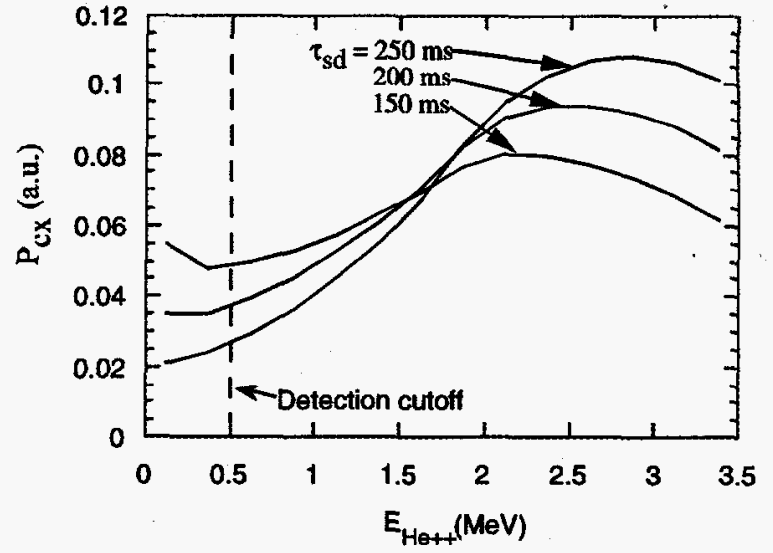

FIG. 27. Calculated probability of charge exchange versus alpha energy. Assuming alphas are lost only on their first charge exchange event, this plot represents the expected energy distribution of charge exchange lost alphas.

as compared to exposure $\mathrm{B}(2.2 \pm 0.3 \mathrm{MeV})$ (see section 4.2.2.) might be explained by the longer slowing down time of the hotter plasma in exposure D (see Table I). At a slowing down time of $200 \mathrm{~ms}, \sim 90 \%$ of alphas are calculated to charge exchange before slowing down below the minimum detection energy of $0.5 \mathrm{MeV}$.

The other potential donors of electrons present in significant quantities are the NBI neutrals [34]. In this case, the velocity of the donor (ie. the neutral beam species) must be taken into consideration since it is an appreciable fraction of the alpha particle velocity. For instance, a $100 \mathrm{KeV}$ deuteron travels at about 1/3 the speed of a 2.0 MeV alpha. The cross section for charge exchange between a fully stripped $2.5 \mathrm{MeV}$ alpha and a neutral $100 \mathrm{KeV}$ deuteron reaches a maximum of $\sim 10^{-18} \mathrm{~cm}^{2}$ when they are traveling in the same direction, and a minimum of $\sim 10^{-21} \mathrm{~cm}^{2}$ when they are traveling in opposite directions [32]. Using the maximum of $10^{-18} \mathrm{~cm}^{2}$ to obtain a conservative estimate, and assuming a density of NBI neutrals of $\sim 10^{9} \mathrm{~cm}^{-3}$ and a beam volume to plasma volume ratio of $\sim 0.01$ to account for the beam localization results in a collision period of $\sim 70 \mathrm{sec}$, making this process insignificant in comparison to impurity charge exchange and slowing down.

The delayed loss observed by the scintillator detectors in DD might be caused by a similar mechanism. Since the DD fusion products are only singly ionized, charge exchange results in neutralization and subsequent straight line trajectories. Reionization in the plasma might allow some of these fusion products to transition to prompt loss orbits [34]. The differing mechanisms might account for the detection of the anomalous loss only in DD plasmas for the scintillator detectors. 


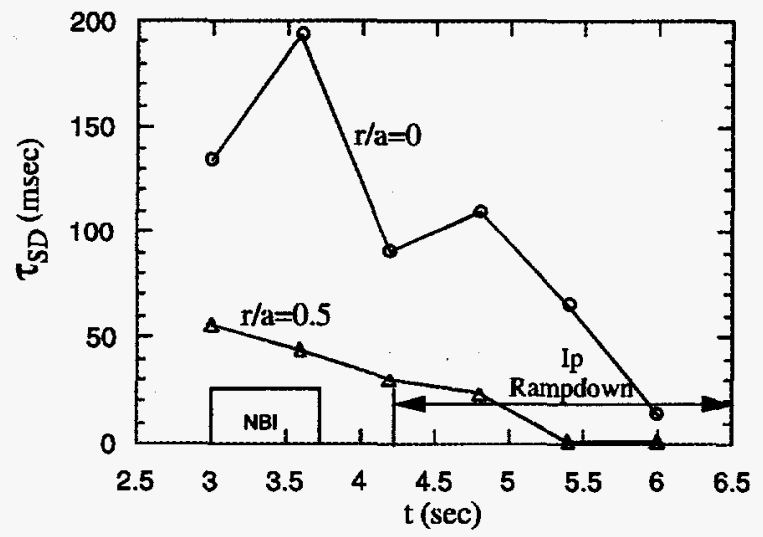

F1G. 28. Slowing down times calculated for $r / a=0$ and $r / a=0.5$ as a function of time in the discharge of exposure $B$.

\subsubsection{Loss during $I_{p}$ rampdown}

As the plasma current is ramped down at the end of a plasma discharge, remaining high energy alphas become deconfined as the downward grad B and curvature drifts become dominant. However, the $I_{p}$ ramp down in exposure B doesn't start until $500 \mathrm{~ms}$ after the end of NBI and since the energy e-folding time $\left(\tau_{s d}\right)$ due to electron drag remains $<200 \mathrm{~ms}$, as shown in Fig. 28, in the plasma core during this time, the alpha energy should have dropped by several factors of $\mathrm{e}$ by the start of the $I_{p}$ ramp. Just 2 e-folding times is sufficient to reduce the alpha energy below the detector's minimum detectable energy of $0.5 \mathrm{MeV}$. The observed peak in the energy distribution occurs at an energy only $\sim 30 \%$ lower than $3.5 \mathrm{MeV}$ and so, assuming there are no accelerating forces acting on the alphas during the ramp down, cannot be attributed to anything that occurs $>100 \mathrm{~ms}$ after the end of NBI. The induced toroidal electric field associated with the $I_{p}$ ramp down can, however, cause a positive acceleration of counter-going passing alphas. But this toroidal acceleration causes them to become more passing, which is not consistent with the detection of co-going particles. Furthermore, if there is a significant lower energy loss occurring after NBI, it should be visible to the lost alpha scintillator detectors but it has never been observed. Therefore, a partially thermalized loss due to the current ramp down at the end of the discharge is not a viable explanation of the anomalous loss data.

It should be mentioned that the $I_{p}$ ramp down in exposure D begins just $200 \mathrm{~ms}$ after the end of NBI which was extended by $300 \mathrm{~ms}$ to increase the total alpha fluence to the collector probe. In section 4.2.2 the peak in the alpha energy distribution was inferred to be $\sim 2.7 \pm 0.3 \mathrm{MeV}$. This is $\sim 0.5 \mathrm{MeV}$ higher than the peak of exposure B which had a $500 \mathrm{~ms}$ delay between the end of NBI and the beginning of the $I_{p}$ ramp down. The higher anomalous loss energy associated with the shorter delay may be an indication that the anomalous loss mechanism does occur after the end of NBI, although, as mentioned above, this is highly unlikely. A scan of the delay time between NBI and the various ramp start times could resolve this issue.

\subsubsection{Scattering off RFL's and collimator walls}

Scattering off of RF limiters and the wall of a collimating port can reduce the energy spectrum of the incoming alphas. However, it is unlikely that scattering could explain the anomalously large alpha fluence observed in the 1.8 MA plasmas since this is not a source of new alphas. It is also unlikely that there will be a significant contribution of scattered alphas in the foil samples since large angle deflections of alphas are quite rare in solids. TRIM-95 simulations (see section 3) of $3.5 \mathrm{MeV}$ alphas implanting into carbon at shallow angles result in just 20\% of the implanted alphas reemerging from the face of a flat piece of carbon when implanted at an angle of incidence of $89^{\circ}\left(1^{\circ}\right.$ grazing angle), and less than $1 \%$ at $85^{\circ}$. In other words, most of the alphas that enter a limiter or the wall of a collimating port will be stopped (within $\sim 11 \mu \mathrm{m}$ ) without reemerging. Bench top implants using aluminum collimators with Am-241 alpha sources were done for calibration purposes. Results showed reasonable agreement to the predicted fluence and range distribution [10]. Furthermore, it is unlikely that the plasma current dependence could be explained by scattering.

\subsubsection{Activation of surrounding materials}

Activation of materials in first wall components by absorption of $14 \mathrm{MeV}$ fusion neutrons and the subsequent release of alphas through $(n, \alpha)$ reactions is very unlikely as a possible explanation of the anomalous loss. The largest cross sections for $(n, \alpha)$ reactions from $14 \mathrm{MeV}$ neutrons are on the order of $10^{-22} \mathrm{~cm}^{2}$ [35]. Using this conservatively large cross section results in a mean free path for a $14 \mathrm{MeV}$ neutron of $\sim 0.1 \mathrm{~cm}$ in solids. Assuming the resulting alphas can escape from within $10 \mu \mathrm{m}$ of the surface, the neutron fluence of $\sim 10^{11}$ neutrons $/ \mathrm{cm}^{2}$ yields $\sim 10^{9}$ alphas $/ \mathrm{cm}^{2}$, or about three orders of magnitude smaller than the levels detected by the alpha collector.

The fact that most of the $(n, \alpha)$ reactions have half lives $>10 \mathrm{sec}$ [35] could allow the collector probe to integrate the alpha collection over a extended duration even after the shot is over, possibly explaining the absence of the anomalous loss on the real time scintillator detectors. However, it would be difficult to explain the pitch angle 
distribution and plasma current dependence of the anomalous loss with such a mechanism.

\subsubsection{Foil surface fusion}

Another possibility that must be considered is that NBI $D \& T$ ions at $100 \mathrm{KeV}$ may be striking the surface of the foil and the walls of the collimating ports and fusing with $D \& T$ that are on the surface. This would give rise to an alpha source with a nearly isotropic velocity distribution near the surface of the foils. These alphas wouldn't have to undergo any collimation so could implant at large incident angles $\left(0^{\circ}\right.$ being normal to the surface) explaining the shallow depth distribution. However, the deeper collimating ports of the redesigned probe head were intended to exclude NBI ions while allowing alphas, which have $\sim 3 \times$ larger gyroradius, to still implant [3]. It can be inferred from the fact that the anomalous loss features remained essentially unchanged between the two designs that foil surface fusion was not significant. This mechanism can also be ruled out on the basis of the current dependence. As $I_{p}$ increases, the confinement of NBI ions should increase, providing less of a source of fusion on the foil surface.

\subsubsection{Diffusion of Residual He}

Residual He in the tokamak, leftover from previous experiments or from alpha ash, will not reach $\mathrm{MeV}$ energies during a discharge with no RF. The first Ni foil layer should stop alphas below $0.5 \mathrm{MeV}$. If the foils are heated sufficiently to allow diffusion of this He into the deeper foil layers there should be a monotonic decrease in the alpha fluence with layer depth from a peak in the shallowest layer. Thus diffusion of residual He can not account for the peaks in the fourth and fifth layers of the 1.8 MA exposures. Furthermore, it is unlikely that the current dependence of the anomalous loss could be explained by diffusion of residual He since the improved confinement associated with the higher plasma current should reduce heating of collector foils. Diffusion of residual He may, however, explain the low energy anomalous loss observed in 1.0 MA discharges.

\subsubsection{Diffusion of Implanted He}

The diffusion of implanted He from layer to layer between the time of implantation and removing the foils from the spool piece cannot explain the anomalously large fluence associated with the 1.8 MA data. Furthermore, if implanted He does diffuse, it should diffuse in both directions and not just toward the shallower layers as implied by the 1.8 MA data.

\section{SUMMARY}

Escaping alpha particles from four DT shots have been collected in stacks of thin nickel foils located within the alpha collector probe on TFTR. The subsequent melting of the foils in a closed volume and measurement of the released $\mathrm{He}$ as a function of layer depth yields a lost alpha energy distribution, with better than $20 \%$ energy resolution [3]. Two rows of eight collimating ports each provide full $360^{\circ}$ pitch angle coverage (with the exception of self-shadowing of very deeply trapped particles by the probe head) with limited pitch angle resolution. The foil deposition technique employed by the alpha collector is accurate to within $\pm 10 \%$ at fluences above $\sim 10^{9}$ alphas, providing an absolutely calibrated measurement to check alpha loss models and to cross calibrate other detection methods. The lack of electronics and optics gives it good immunity to high neutron fluxes, and the use of a mass spectrometer in the analysis allows for positive ion identification. Although these features make the alpha collector an attractive diagnostic for future fusion reactors, several disadvantages may make other methods preferable. For instance, the alpha collector has no intrinsic time resolution, requires accessibility to retrieve the exposed foils, and requires a long turn around time between exposure and analysis.

The alpha collector has been used to measure escaping alphas in $2.45 \mathrm{~m}$ plasmas for two discharges at a plasma current of 1.0 MA, and two discharges at 1.8 MA. For the 1.0 MA discharges, the total alpha fluence, energy distribution, pitch angle distribution, and radial distribution are all in good agreement with the first orbit loss model, and with the signals from the nearby $90^{\circ}$ lost alpha scintillator detector, with the exception of a small anomalous loss feature at an energy below $\sim 2 \mathrm{MeV}$. The results of the 1.8 MA discharges, however, display a large anomalous loss feature, in addition to first orbit loss, with an alpha fluence a factor of $\sim 6$ larger than predicted by the first orbit loss model. This anomalous loss is broadly peaked at an energy of $\sim 2.5 \mathrm{MeV}$. From this partial thermalization it can be inferred that this loss is 'delayed' with respect to alpha production by about one third of the energy efolding time. The anomalous loss occurs for particles that are co-going at the detector which are more trapped than the fattest banana orbit, and it exhibits a strong radial dependence which may be due to RF limiter shadowing, or an indication of a diffusive loss mechanism. The signals of the $90^{\circ}$ scintillator detector during these discharges, however, are in agreement with the first orbit loss model and do not display any sign of this anomalous loss.

The qualitative characteristics of the anomalous loss detected at 1.8 MA with the alpha collector probe are con- 
sistent with those of the 'delayed' loss feature identified in DD plasmas with the $90^{\circ}$ scintillator detector [17]. This implies that they may be due to the same loss mechanism, although, it is not understood why the anomalous loss does not appear on the scintillator detectors in DT.

The strong radial dependence of the anomalous loss, a factor of 3 decrease in measured fluence from the upper to the lower row of collimating ports, suggests a radial diffusive process, which should cause a reduction in alpha flux due to scrape-off outside the radius of an obstacle such as the RF limters. However, to go from a marginally confined orbit, which just misses the outer midplane, to the anomalous loss orbit which intercepts the detector, the alpha banana tip would have to jump at least $15 \mathrm{~cm}$. Such a large step-size results in very little radial variation over the $1.1 \mathrm{~cm}$ separation between the upper and lower rows of collimating ports. If it is assumed that orbits exist that intercept the RF limiters at the bottom of the vessel first, rather than at the midplane, than a small step-size of $\sim 0.2 \mathrm{~cm}$ can account for the factor of 3 drop. However such orbits require that the alpha energy be below the minimum detectable energy of $\sim 0.5 \mathrm{MeV}$. Thus there appears to be an inconsistency between the radial dependence and the diffusive step-size required to bring an alpha to the detector, making it difficult to develop a model which accounts for the characteristics of the anomalous loss. The other possibility is that the lower row is partially in the shadow of the nearby RF limiter. A scan in the radial position of the probe would allow a conclusive result.

Several possible mechanisms have been considered in an attempt to explain the anomalous loss. The explanation that is most consistent with the observations is charge exchange loss, in which previously confined alpha orbits transition to prompt loss orbits as a result of electron capture from $\mathbf{H}$-like carbon impurities. Further work is needed to quantify this loss mechanism and to determine if its effects should be evident to other escaping and confined alpha diagnostics. The most obvious approach is to develop a guiding center Monte Carlo code to take into account the probabilities for charge exchange and reionization as a function of alpha energy and minor radius (since plasma and impurity density depend on minor radius). However, the absence of the anomalous loss on the scintillator detectors and its radial dependence do not appear to be consistent with the large step-size diffusion that would be associated with this loss process.

In the design of a fusion reactor it is necessary to be able to predict the alpha wall loading in order to prevent hot spots. Thus, the global alpha loss and its distribution to the first wall is more important than the loss to a localized detector. Unfortunately, it is difficult to make wall loading predictions based on the loss to a single detector. This is because the effective wall area (ie. the area generated by projecting the trajectories of the detected particles onto the first wall as if they had not been stopped by the detector) of a given detector may vary widely between different types of alpha loss. Although the detected anomalous loss is significantly larger than the predicted first orbit loss, this loss may be preferentially concentrated in the detector (due to a large effective wall area), resulting in a lower actual wall loading than might be expected. For instance, for a small-step diffusive loss mechanism, the effective wall area generated by a detector positioned inside the $R F$ limiter radius can be relatively large since the detector can scrape-off particles that would otherwise have spread over a large area owing to the randomization of the diffusion process.

Similarly, without knowing the loss mechanism, it is difficult to estimate the global alpha loss based on a localized detector since this measurement provides no information regarding poloidal distribution. Assuming that the mechanism responsible for the anomalous loss is impurity charge exchange, then the gc-ORBIT code estimate of $\sim 20 \%$ global loss, which is $\sim 8$ times the first orbit loss prediction (see section 5.4.4), can be taken as a 'best guess' of the upper limit of the global anomalous alpha loss. However, this may be a gross over-estimate due to the potentially large uncertainties in the charge exchange cross section and the method used to quantify this loss mechanism. As of yet, no other diagnostic results have suggested the existence of such a loss. As for a lower limit of the global anomalous alpha loss, it is conceivable that this loss is very poloidally localized to the bottom of the vessel or that it is concentrated in the detector (due to a large effective wall area), resulting in an insignificant global loss in comparison to first orbit loss.

Further work might help in understanding the significance of this anomalous loss. Further modeling, particularly of the charge exchange loss mechanism, might provide an explanation for the anomalous loss. Scans in plasma current, major radius, beam power, and radial position could shed more light on the anomalous loss. And probe head design changes could improve the quality of the measurement. Such changes could include narrower collimating ports to improve pitch angle resolution, thinner nickel foils to improve energy resolution, and programmable shutters over the foils to improve time resolution.

\section{ACKNOWLEDGMENTS}

The authors thank G. Lemunyan, M. Vocaturo, A. Brooks, D. Loesser, B. LeBlanc, K. Owens, D. John- 
son, $\mathrm{K}$. Young, $\mathrm{K}$. McGuire and R. Hawryluk as well as the Health Physics Division at PPPL for their assistance and support in the development of this diagnostic. We also thank E. Synakowski, K. Hill, M. Herrmann, and R. Heeter of PPPL, and T. Winter of Penn State for many useful discussions. This work was supported by US DoE contract number DE-AC02-76-CHO-3073, Canadian Fusion Fuels Technology Project, and Natural Sciences and Engineering Research Council of Canada

\section{REFERENCES}

[1] ZWEBEN, S.J., et al., Nucl. Fusion 35 (1995) 893

[2] DARROW, D.S., et al., Rev. Sci. Instrum. 66 (1995) 476

[3] HERRMANN, H.W., et al., Rev. Sci. Instrum. 66 (1995) 351

[4] HERRMANN, H.W., Anomalous Loss of DT Alphas in TFTR, Thesis to be submitted for Ph.D., Princeton University, 1997

[5] LANGLEY, R.A., Bull. Am. Phys. Soc. 29 (1984) 1309

[6] CARRUTHERS, E.V., et al, J. Nucl. Mater. 176/177 (1990) 1027

[7] ZHU, J., et al., Nucl. Instr. and Meth. B59/60 (1991) 168

[8] ZIEGLER, J. F., BIERSACK, J.P., LITTMARK, U., The Stopping and Range of Ions in Solids, Pergamon Press, Oxford (1985)

[9] CHONG, G.P., et al., Bull. Am. Phys. Soc. 39 (1994) 1675

[10] CHONG, G.P., Measurement of Helium in Nickel Foils from the TFTR Alpha Collector Probe, Masters of Applied Science Thesis, University of Toronto Institute for Aerospace Studies, 1995

[11] REDI, et al., Nucl. Fusion 35 (1995) 1191

[12] ZWEBEN, S.J., et al., Nucl. Fusion 30 (1990) 1551

[13] HEIDBRINK, W.W. et al., Nucl. Fusion 34 (1994) 535

[14] FELT, J., et al, Rev. Sci. Instrum. 61 (1990) 3262

[15] HAWRYLUK, R., et al., in Physics of Plasmas Close to Thermonuclear Conditions (Proc. Course Varenna, 1979), Vol. 1, CEC, Brussels (1980) 19

[16] CHOE, W., Princeton Plasma Physics Laboratory, personal commuication (1996)

[17] ZWEBEN; S.J., et al., Nucl. Fusion 33 (1993) 705

[18] ZWEBEN, S.J., et al., Rev. Sci. Instrum. 63 (1992) 4565

[19] BOIVIN, R.L., et al., Nucl. Fusion 33 (1993) 449

[20] WANG, S.T., ZWEBEN, S.J, Model of Alpha Particle Diffusion in the Outer Limiter Shadow of TFTR, PPPL Report 3186, Princeton Plasma Physics Laboratory (1996), submitted to Nucl. Fusion Lett.

[21] ZWEBEN, S.J., et al., Phys. Plasmas 1 (1994) 1469

[22] CHANG, C.S., et al., Phys. Plasmas 1 (1994) 3857

[23] GOLDSTON, R.J., et al., Phys. Rev. Lett. 47 (1983) 647

[24] WHITE, R.B., Phys. Fluids B 2 (1990) 845

[25] ZWEBEN, S.J., et al., 14th Int. Conf. on Plasma Physics and Controlled Nuclear Fusion Research, Wurzburg, 1992, IAEA, Vienna, Vol.1 (1993) 363

[26] MYNICK, H.E., in Theory of Fusion Plasmas (Proc.
Joint Varenna-Lausanne Int. Workshop Chexbres, 1988) Editrice Compositori, Bologna (1988) 385

[27] OLSON, R.E., Phys. Lett. 71A (1979) 341

[28] HULSE, R.A., Nucl. Technol. Fusion 3 (1983) 259

[29] OLSON, R.E., Phys. Rev. A16 (1977) 531

[30] WINTER, T.G., Phys. Rev A47 (1993) 264

[31] WINTER, T.G., Pennsylvania State University, WilkesBarre Campus, personal communication (1996)

[32] PHANEUF, R.A., et al., Atomic Data for Fusion, Vol. V, ORNL-6090 (1987)

[33] BARNETT, C.F., Atomic Data for Fusion, Vol. I, ORNL$6086(1990)$

[34] HEETER, R.F., Bull. Am. Phys. Soc. 40 (1995) 1768

[35] International Atomic Energy Agency, Handbook on Nuclear Activation Data, Tech. Report Series 273, IAEA, Vienna (1987) 261-304 